\title{
EEG and behavioural correlates of different forms of motor imagery during action observation in rhythmical actions
}

\author{
Eaves, D. L. ${ }^{a, b^{*}}$, Behmer Jr., L. P. ${ }^{c, d *} \&$ Vogt, S. ${ }^{a^{*}}$ \\ ${ }^{a}$ Department of Psychology, Fylde College, \\ Lancaster University, Lancaster, LA1 4YF, UK \\ ${ }^{\mathrm{b}}$ Sport and Exercise Science Section, \\ Teesside University, Middlesbrough, TS1 3BA, UK \\ ${ }^{\mathrm{c}}$ Department of Psychology, Washington State University, \\ Pullman WA, 99164-4820, USA \\ ${ }^{\mathrm{d}}$ Department of Psychology, Brooklyn College \\ New York, New York 11210, USA
}

Corresponding author - pre-publication:

*Daniel L. Eaves e-mail: d.eaves@tees.ac.uk

Corresponding authors - post-publication:

*Daniel L. Eaves e-mail: d.eaves@tees.ac.uk

*Lawrence Behmer e-mail: lawrencebehmer@gmail.com

*Stefan Vogt e-mail: s.vogt@lancaster.ac.uk 


\begin{abstract}
Recent studies show that participants can engage in motor imagery (MI) and action observation (AO) simultaneously (AO+MI), indicating a capacity for 'dual action' simulation. Here we studied the electrophysiological correlates and behavioural outcomes of two forms of AO+MI, along with pure $\mathrm{MI}$ and pure AO control conditions. In synchronised $\mathrm{AO}+\mathrm{MI}$, participants imagined performing a rhythmical action in synchrony with an observed distractor action. In contrast, in static AO+MI, they imagined holding a static hand posture during AO. Following synchronised $\mathrm{AO}+\mathrm{MI}$, rhythmical execution was strongly biased towards the cycle time of the previously observed rhythm ('imitation bias'), whereas a weaker bias was found following pure MI, and particularly for static AO+MI. In line with these findings, event-related desynchronisation (ERD) in primary sensorimotor and parietal regions was more pronounced in synchronised $\mathrm{AO}+\mathrm{MI}$ compared to both pure $\mathrm{AO}$ and pure MI. These ERD amplitudes were, however, highly similar for static and synchronised $\mathrm{AO}+\mathrm{MI}$; suggesting that, regardless of co-represented content, both $\mathrm{AO}+\mathrm{MI}$ states produced stronger motor activations than single action simulation. In contrast, synchronised AO+MI produced significantly stronger ERD in rostral prefrontal cortex compared to the other three conditions. This specific rostral prefrontal involvement most likely reflected additional cognitive processing for aligning dual action simulations. Together these results provide an important empirical validation of different $\mathrm{AO}+\mathrm{MI}$ states, in that the imitation bias was strongly modulated by the content of the $\mathrm{AO}+\mathrm{MI}$ instructions, and that synchronised $\mathrm{AO}+\mathrm{MI}$ produced stronger behavioural and neurophysiological effects compared to pure AO or MI.
\end{abstract}

Key words: mental practice; mirror neuron system; motor simulation; observational practice; action observation network; observational learning; mental rehearsal 


\section{Introduction}

Action observation (AO) and motor imagery (MI) can both be regarded as sub-forms of motor simulation that involve the motor system but do not include actual motor execution (Jeannerod, 1994, 2001, 2006). Despite this early integrative account, AO and MI have, up until recently, largely been studied in isolation from each other (Vogt et al., 2013). One important and seemingly overlooked issue is the possibility that participants can engage in $\mathrm{AO}$ and $\mathrm{MI}$ simultaneously ('AO+MI'), wherein their contents can coincide, complement each other, or compete (ibid.). In a recent behavioural study (Eaves et al., 2014), we indeed demonstrated that the particular contents of MI during AO can substantially modulate automatic imitation effects in movement kinematics. The experimental paradigm employed thus proved a useful tool for studying dual motor representations. In the present study we used the same paradigm to explore the neural correlates of two different AO+MI states (synchronised vs. static, see below), compared with both pure AO and pure MI. Our primary focus was on the related brain activity using electroencephalography (EEG), and we also report the automatic imitation effect as an 'imitation bias' in subsequent movement kinematics. Next we describe the background of the present research, followed by the aims of both the behavioural and electrophysiological analyses.

Previously we showed that observing a task-irrelevant rhythmical action, in either a fast or slow pace, during a motor preparatory phase significantly biased the cycle time of a subsequently executed rhythmical action (Eaves et al., 2012). We interpreted this as an instance of visuomotor priming (Vogt \& Thomaschke, 2007) or automatic imitation (Heyes, 2011). Whilst most related studies had used reaction times, by studying rhythmic alignment we were able to quantify the similarity between the to-be-executed 'instructed action' and the passively observed distractor action as an imitation bias. In addition, since we used an appropriately long time window (4 sec) for distractor observation, processing the observed action could interact with motor preparation of the instructed action in real time. Based on the framework by Cisek and Kalaska (2010), we conceptualised these two processes as concurrent and potentially competing action simulations. The imitation bias was indeed stronger when the two actions fully matched, compared to when either the action goal or plane differed. When both dimensions were incompatible, the bias was not reduced further, however. We interpreted these results in favour of an integrated account, where the usefulness of the observed distractor for supporting motor planning would influence the extent to which the two sensorimotor streams would either merge or compete with one another (Cisek and Kalaska, 2010). 
In the follow-up study (Eaves et al., 2014) we aimed to manipulate the competition between these two hypothetical sensorimotor streams further by instructing different forms of MI during AO. Participants imagined performing the instructed action either in synchrony with the observed action ('synchronised $\mathrm{AO}+\mathrm{MI}$ '), or they imagined performing a static hand posture during $\mathrm{AO}$ ('static $\mathrm{AO}+\mathrm{MI}$ '). Importantly, the imitation bias was significantly stronger following synchronised AO+MI compared to passive AO. Moreover, this bias was not modulated by the compatibility between the contents of AO and MI, which varied in action type, plane of motion, or both. That is, these coordinative AO+MI conditions also enhanced the imitation bias. In contrast, static AO+MI practically abolished the imitation bias. Accordingly, this study provided the first empirical evidence for differential effects of specific $\mathrm{AO}+\mathrm{MI}$ states, ranging from congruent, across coordinative, to conflicting $\mathrm{AO}+\mathrm{MI}$, respectively (Vogt et al., 2013).

In the present study we sought to investigate this dual-simulation account of AO+MI further, using both behavioural and electrophysiological data. We focussed on two largely unexplored issues. The first was the assumption that in $\mathrm{AO}+\mathrm{MI}$ states, the $\mathrm{AO}$ and $\mathrm{MI}$ component processes operate essentially in an additive manner. Here we were primarily interested if synchronised AO+MI would exert both stronger behavioural and electrophysiological effects when compared with pure MI.

The second objective was an in-depth comparison between synchronised and static AO+MI. To start with, we sought to replicate the contrasting behavioural effects of synchronised vs. static AO+MI as observed in Eaves et al. (2014). Our main interest here, however, was in the electrophysiological correlates of these two AO+MI states, which have not been contrasted before. One possibility was that the EEG data would essentially mirror the contrasting behavioural results, that is, synchronised $\mathrm{AO}+\mathrm{MI}$ would exert stronger neural activations than static AO+MI. Alternatively, both AO+MI states might evoke similar cortical activity, which might be predicted on the basis that both involve dual action representations. We now describe a series of more specific aims for both the behavioural and electrophysiological analyses separately, while referring to previous literature where appropriate. Finally, we expand our predictions further for our second core aim stated above, that is, to contrast the two AO+MI states directly (i.e., aim 2.4 below).

\section{Aims of the behavioural analysis}

While in our previous behavioural studies we compared the imitation bias for different AO+MI states to the bias from 'passive' AO (Eaves et al., 2014), here we extend the 
comparisons to include pure MI. Thus our first behavioural aim (1.1) was to investigate if a bias can be obtained in rhythmical action execution, following pure MI at different speeds (fast vs. slow) across trials. While earlier research shows MI content can modulate response initiation times in subsequent reach-grasp movements (Ramsey et al., 2010), we sought fresh evidence in movement kinematics.

The second and equally under-researched aim (1.2) of the behavioural analysis was to compare the effects of pure MI to synchronised AO+MI. It is at least conceivable that the motor imagery component of $\mathrm{AO}+\mathrm{MI}$ procedures is the main or sole driver of the behavioural effects. However, given the substantial evidence for visuo-motor priming effects (for reviews see Heyes, 2011; Vogt \& Thomaschke, 2007), which are most likely unmediated by motor imagery (e.g., Vogt, 1996), in the present study we expected stronger effects for AO+MI than for pure MI. Finally, as a crucial prerequisite for the electrophysiological analyses, the third aim (1.3) was to replicate our earlier finding that the imitation bias was substantially stronger for synchronised $\mathrm{AO}+\mathrm{MI}$, compared to static $\mathrm{AO}+\mathrm{MI}$. Note in our pure $\mathrm{AO}$ condition, participants did not subsequently execute the instructed action, thus no behavioural data were available for this condition.

In summary we pursued the following three aims in the behavioural analysis. We predicted the imitation bias would be:

1.1 Present in the pure MI condition;

1.2 Stronger for synchronised AO+MI, compared to pure MI;

1.3 Stronger for synchronised AO+MI, compared to static AO+MI

\section{Aims of the electrophysiological analysis}

In contrast to the behavioural analysis of motor execution, which followed motor simulation, the electrophysiological analyses were concerned with the immediate correlates of the motor simulation states. In EEG recordings a decrease in spectral power from baseline is interpreted as an increase in event-related cortical activity or arousal (event-related desynchronization; ERD), whereas an increase in spectral power from baseline indicates a resting state or idling of cortical neural activity (event-related synchronisation; ERS; Neuper et al., 2006; Pfurtscheller \& Lopes da Silva, 1999; Pfurtscheller et al., 1996). Several papers report significantly greater than baseline mu and beta ERD over the motor cortex during both AO (Avanzini et al., 2012; Calmels et al., 2006; Cochin et al., 1998; Cochin et al., 1999; Cevallos et al., 2015) and MI (Pfurtscheller et al., 2006; Pfurtscheller \& Neuper, 1997). Accordingly, we analysed ERD in the mu and beta bands, which can be taken to reflect downstream signals 
from premotor regions (e.g., Behmer \& Jantzen, 2011; Beisteiner et al., 1995; Lang et al., 1996; Muthukumaraswamy et al., 2004; Wang et al., 2004; see Pineda, 2005). That is, we used ERD as a marker of premotor and/or motor cortical involvement during the different motor simulation states.

Our first basic aim in the electrophysiological analysis (2.1) was to confirm that all four conditions increased neural activity in primary sensorimotor and parietal areas, relative to a pre-stimulus baseline. Our second aim (2.2) was to compare synchronised AO+MI to (a) pure AO and (b) pure MI. A growing number of neuroimaging studies have already shown that observing whilst imagining the same action (i.e., congruent AO+MI) can yield stronger activations in cortical motor regions, compared to pure AO (Berends et al., 2013; Macuga \& Frey, 2012; Mouthon et al., 2015; Nedelko et al., 2012; Taube et al., 2015; Villiger et al., 2013; Wright et al., 2014). In contrast, AO+MI and pure MI were compared directly in four studies (see Mouthon et al., 2015; Neuper et al., 2009; Taube et al., 2015; Wright et al., 2014), with less clear-cut results (see Discussion). Therefore, we were particularly interested if there would be stronger neural activity during synchronised $\mathrm{AO}+\mathrm{MI}$ than during pure $\mathrm{MI}$ (aim 2.2b), which would match our related prediction for the behavioural data (i.e., aim 1.2). We also compared ERD intensities directly between pure AO and pure MI (aim 2.3).

As stated earlier, a core objective in the present study was the contrast between ERD for synchronised $\mathrm{AO}+\mathrm{MI}$ against static $\mathrm{AO}+\mathrm{MI}$ (aim 2.4), which has not previously been studied. Synchronised AO+MI requires tight temporal couplings between the two sensorimotor representations, whereas static $\mathrm{AO}+\mathrm{MI}$ requires participants to focus more unilaterally on their motor imagery, and even resist synchronising this with the observed action. Whilst one might expect sensorimotor activations would be stronger in either synchronised AO+MI (e.g., due to an additive effect of merging the two action representations) or in static $\mathrm{AO}+\mathrm{MI}$ (e.g., due to a dominant effect of inhibitory MI processes over $\mathrm{AO}$ ), this motor-related activity might also be equally pronounced across the two conditions, since both involved dual-action simulation. However, in the latter case differences between synchronised and static AO+MI might still be expected in other brain regions.

In summary, in the EEG data, we predicted significantly stronger ERD for:

2.1 Each of the four experimental conditions (i.e., pure AO, pure MI, synchronised $\mathrm{AO}+\mathrm{MI}$, and static $\mathrm{AO}+\mathrm{MI})$, compared to the pre-stimulus baseline;

2.2 Synchronised AO+MI, compared to both: (a) pure AO, and (b) pure MI;

2.3 We also compared ERD for pure AO to pure MI; 
2.4 Finally, we investigated the ERD for synchronised AO+MI, compared to static AO+MI.

\section{Materials and Methods}

\subsection{Participants}

Fourteen participants ( 7 female, mean age $28.6 \mathrm{yrs} ; S D=5.5 \mathrm{yrs}$ ) volunteered, with normal ( $n$ $=5$ ) or corrected-to-normal vision. Participants were naïve to the study's purpose, right-hand dominant (Edinburgh Handedness Inventory: $M=97$ Oldfield, 1971) and without physical injuries. We obtained written informed consent prior to their participation, and ethical approval had been granted by Teesside University.

\subsection{Task and Design}

The four main experimental conditions were pure AO, pure MI, synchronised AO+MI, and static $\mathrm{AO}+\mathrm{MI}$. The events in the first two conditions were designed to match the AO+MI conditions as closely as possible. In the 'pure $A O$ ' condition participants verbally reported the match (i.e., same or different) between two actions (for details, see Section 2.4.2). First they saw a picture of a model performing a rhythmical action (with no specific instruction in this condition), followed immediately by a short movie of the same model pantomiming the same action at either a fast or slow pace ('distractor action'). Finally they saw another picture of the model pantomiming either the same or a different rhythmical action. Across trials these actions were either painting or face washing in either the horizontal or vertical plane (see Figure 1 and Table 1).

In the 'pure MI' condition participants briefly observed a movie of the model pantomiming the same actions as in pure $\mathrm{AO}$, at either a fast or slow pace across trials ('instructed action'). They then imagined performing this action at the speed observed in the previous movie, before executing the action at their own preferred pace.

In both AO+MI conditions participants saw a picture of the instructed rhythmical action, followed by a short movie of the model pantomiming either the same or a different action at either a fast or slow pace across trials ('distractor action'). During the movie, participants always imagined performing the instructed action either in synchrony with the action in the movie 'synchronised $A O+M I$ ', or as a static posture 'static $A O+M I$ ', before executing the instructed action at their own preferred pace. 
--- Insert Table 1 about here ---

We used a three-factorial repeated-measures design. Each of the four experimental conditions (pure AO, pure MI, synchronised AO+MI, or static AO+MI) consisted of sixtyfour trials, each split into two sub-blocks of 32 trials. On the main day of testing we first ran the four conditions as four sub-blocks in a counterbalanced order across participants, followed by the four remaining sub-blocks in the same order for each participant.

The other two factors were distractor speed and the compatibility between instructed and distractor actions: both were manipulated within each sub-block. As in our two previous studies, the speed of the distractor movie was either fast or slow (4 s of either 60 or 90 beats per min), and the effect of this manipulation on the subsequent execution across trials was measured as the imitation bias (note: for pure MI this factor refers to the speed of the instructed action movie).

While the compatibility manipulation was crucial in our previous behavioural studies, here we only assessed this factor in the behavioural data for both AO+MI conditions. In the EEG analyses, however, we collapsed across this factor. Combining the two individual compatibility manipulations yielded one compatibility factor with four levels: SA/SP, SA/DP, DA/SP or DA/DP. See Footnote 1.

Half of the trials in each sub-block were fully-compatible (i.e., $n=16$ ), while the other half were incompatible. We used an even mix of the three incompatible conditions, with one extra DA/DP trial needed to make up the required 16 incompatible trials in each subblock. Within each block we presented the distractor speeds and the compatibility levels, along with the associated action type and plane combinations, in a quasi-random order.

\subsection{Stimuli and apparatus}

We used a digital video camera (Panasonic NV-MX500B) to create the action pictures and movies. We used two instructed action types (face-orientated: face washing, and surfaceorientated: painting; see Figure 1). Since each action was also instructed to be in either the horizontal or vertical plane, this provided four instructed actions. The model performed all actions with her left hand to provide mirror images of the participants' actions, who always executed actions with their right hand. This arrangement provided spatial compatibility between displayed and performed actions, which can facilitate imitation, relative to an 
anatomically-matched but spatially-incompatible arrangement (e.g., Buccino et al., 2004; Koski et al., 2003).

We used eight distractor movies, one slow and one fast version of the four instructed actions. While the model's performance was paced by a metronome to achieve the exact distractor speeds shown in Table 1, we displayed all stimuli without sound. Note in each picture of the instructed action the model held the relevant object (sponge or paintbrush) to allow quick discrimination between actions. Participants pantomimed their actions (i.e., without objects) so they did not need to select an object for each trial.

We showed pantomimed actions in the distractor movies so participants could distinguish between instructed and distractor stimuli, and to potentially strengthen the impact of the distractor on their pantomimed execution. In the pure AO condition we displayed the final action picture without an object (i.e., a pantomime action) to encourage comparison with the distractor movie, rather than with the instructed action picture.

Participants sat at a wooden desk facing a 17-in LCD computer monitor (Apple Studio Display) positioned $80 \mathrm{~cm}$ away from their head. All stimuli were displayed against a black background via PsyScript 2.3 software (http://www.lancaster.ac.uk/psychology/research/research-software/psyscript2/) running on a Power Macintosh G4 computer fitted with a digital I/O board.

The start location for the participants' right index finger and thumb was on an electroconductive plate mounted on top of a $23 \mathrm{~cm}$-tall wooden post, $20 \mathrm{~cm}$ in front of them on the desk. This start position was equidistant from the workspaces needed to execute each action. A magnetic motion sensor was fitted to the distal end of the second metacarpal bone of the right hand. Participants' kinematic data were sampled at $103 \mathrm{~Hz}$ in 3-D space for $4 \mathrm{~s}$ periods using a Minibird Magnetic Tracking System (Ascension Technology Corp.), and stored on a separate PC. At the end of each trial, kinematic data plots were displayed on a second monitor, unseen by participants.

\subsection{Procedures}

\subsubsection{Familiarisation}

We conducted the main familiarisation procedures (Phases 1-5) on Day 1, while all of the main experimental procedures were completed on the consecutive Day 2. In Phase 1 participants learned to pantomime each action from a set of four familiarisation movies (four actions with four attempts each). These movies were the same as those in the main 
experiment, except the cycle times were mid-way between the two distractor speeds shown in Table 1 (i.e., 75 bpm).

We gave participants verbal feedback about their movement based on the kinematic plots. This ensured their movement amplitude and cycle time aligned with the medium-paced stimuli. In Phase 2, participants saw a picture of each action while they pantomimed the same action for $4 \mathrm{~s}$ ( 8 trials). In Phase 3, they experienced the basic trial structure for the two $\mathrm{AO}+\mathrm{MI}$ conditions in the main experiment, including all associated compatibility conditions (16 trials).

Learning is a key component of the PETTLEP model for mental imagery training (see Holmes \& Collins, 2001). Therefore, in Phase 4 we introduced participants to MI via a modified version of the Movement Imagery Questionnaire-3 (MIQ-3; Williams et al., 2012). While we retained the arm extension action from the original MIQ-3, we adapted the questionnaire to include only single arm actions, similar to those in our experiment (i.e., cuplifting and pantomime tooth-brushing).

Participants performed an overt followed by an imagined version of each action. They then self-reported the vividness of each experience on three subscales: visual internal, visual external and kinaesthetic imagery (3 actions per subscale; mean scores = 5.7, 4.2 and 5.8 / 7, respectively). We then read an MI script designed using the PETTLEP principles. This provided a $1^{\text {st }}$ person (internal), kinaesthetic MI experience of the two instructed action types used in the main experiment.

In Phase 5, participants practiced the trial structure for the four experimental conditions: First, synchronised AO+MI, then static AO+MI (16 trials each); followed by pure MI and then pure AO (8 trials each). We gave verbal feedback if movements drifted away from the criterion cycle time (i.e., $75 \mathrm{bps})$ or amplitude $(10 \mathrm{~cm})$. Shortened versions of these five phases were run at the beginning of Day 2.

\subsubsection{Main experiment}

Our core manipulation across trials was that of distractor speed, with a slow:fast ratio of $150 \%$ (see Table 1). We did not inform participants about these subtle speed changes. For all four experimental conditions, the main period of interest for EEG data collection was Event B (Figure 2), whereas the main period of interest for kinematic data collection was Event C (note that pure AO did not involve motor execution). We next describe the task differences between the four experimental conditions. 
--- Insert Figure 2 about here ---

Pure AO. In this condition, participants started each trial by pressing the space bar on a keyboard. As in the other three conditions, a white fixation cross was then displayed for $1.5 \mathrm{~s}$ (baseline), followed by a green circle (1s 'get ready' cue; see Figure 2). Participants were then shown a picture of the instructed action for $2 \mathrm{~s}$ (Event A, with no specific instruction in this condition), followed by a distractor movie of the same actor pantomiming the same rhythmical action for 4s (Event B). Upon distractor movie offset a black screen preceded onset of the final action picture (Event C). Participants then verbally reported whether the final action matched the preceding distractor action, and then started the next trial. This task ensured participants paid attention to the distractor action. Participants were not required to prepare or execute an action in this condition in order to minimise any possible motor cortical involvement during distractor action observation. We also asked participants: 'Please refrain from undertaking any MI during distractor observation in this pure AO condition.'

Pure MI. As in the two AO+MI conditions, participants began each trial by placing their right forefinger on top of the wooden post. Following the presentation of the fixation cross and green circle, in Event A participants then observed a movie of the to-bepantomimed 'instructed action' for $2 \mathrm{~s}$, followed by a purple fixation cross for $4 \mathrm{~s}$ (Event B). During this event, they imagined from a $1^{\text {st }}$ person (internal) perspective the physical sensation and effort involved in performing the instructed action with their right hand at the same pace as shown in the preceding movie. The appearance of a black screen (Event $\mathrm{C}$ ) then cued motor execution of the instructed action at their own preferred cycle time for $4 \mathrm{~s}$, during which kinematic data were recorded. As in the two AO+MI conditions, a computer-generated auditory tone signalled the end of this period, whereupon participants returned to the start position to begin the next trial.

Synchronised and static $A O+M I$. In Event A for both of these two conditions, participants observed a picture of the to-be-pantomimed instructed action for $2 \mathrm{~s}$, followed by a distractor movie showing either the same or a different action for $4 \mathrm{~s}$ in Event $\mathrm{B}$. As in the study by Eaves et al. (2014) for synchronised $A O+M I$ participants imagined from a $1^{\text {st }}$ person perspective the physical sensations and effort involved in performing a dynamic version of the instructed action, in synchrony with the displayed rhythmical distractor action. For static $A O+M I$ they imagined their right hand in the static posture of the instructed action, again from a $1^{\text {st }}$ person perspective and with the physical sensations and effort involved when 
adopting this posture, while observing the rhythmical distractor movie. In both AO+MI conditions participants then executed the instructed action at their own preferred pace for $4 \mathrm{~s}$ (Event C) whilst kinematics were recorded. We ensured participants attended to the distractor in the AO+MI conditions by asking them to verbally report both the match between the instructed and distractor actions (same or different), and the distractor properties (action type and plane of motion) on $10 \%$ of all trials in a pseudo-random order. While we did not optimise the experiment to assess the compatibility manipulation between instructed and distractor actions in the EEG analysis (where we collapsed across this factor), we did assess this factor in the behavioural data for the two AO+MI conditions.

In all conditions we asked participants to visually fixate on the model's left eye when observing the rhythmical action stimuli. This prevented them from coupling their eye movements to the model's rhythmical arm movements (i.e., avoiding sensorimotor synchronisation effects, c.f., Schmidt et al., 2007). We also provided a single 'warm-up' trial before each block, and gave short rests between blocks. We included a 10 min rest between the two sets of four sub-blocks to reduce possible effects of fatigue.

\subsection{Data Analysis}

\subsubsection{Behavioural data}

We calculated mean cycle times (in ms) between peak minimum kinematic positions using a customised signal processing application created in Microsoft Visual Studio. First, a $6 \mathrm{~Hz}$ low-pass, $2^{\text {nd }}$ order, bi-directional Butterworth filter smoothed the data. For both horizontal and vertical actions, the first data point was the first peak minimum. This meant we did not analyse hand movements during the initial spatial positioning phase for each action. We calculated mean cycle time across all peak minimum positions available within a $2 \mathrm{~s}$ time window, which involved either two or three cycles. We discarded all trials with erroneous responses (incorrect or no action; $n=12$ ).

Our two dependent measures were the mean response cycle times (ms) and the mean cycle time ratios (\%) between slow and fast trials. Regarding our aim 1.1, we focused the analysis of the mean cycle time (ms) data on the within-subjects factor of distractor speed (slow vs. fast), as this factor was only available for this measure (for pure MI this factor refers to the speed of the instructed action movie). Accordingly, we ran a two-factorial ANOVA on the ms data (involving the factors experimental condition and distractor speed), followed by simple main effect analyses to investigate if the imitation bias was present in the 
three experimental conditions involving execution (i.e., pure MI, synchronised AO+MO, and static $\mathrm{AO}+\mathrm{MI})$.

For aim 1.2 we compared the imitation bias for synchronised AO+MI to pure MI using a one-sample t-test on the cycle time ratio data (\%). For aim 1.3 we used a twofactorial, repeated-measures ANOVA to assess the factors experimental condition ( 2 levels: synchronised AO+MI or static AO+MI) and compatibility (4 levels: SA/SP, SA/DP, DA/SP or DA/DP).

We recorded reaction time data to identify trials with anticipatory ( $<200 \mathrm{~ms} ; n=3)$ or omission errors (>1300 ms; $n=19)$, which we discarded from all analyses. In total, we only removed $1.3 \%$ of trials from the behavioural analyses.

\subsubsection{EEG data}

We used 128 EEG channels sampling at $250 \mathrm{~Hz}$, with an analogue hardware band pass filter at 0.1 to $100 \mathrm{~Hz}$ (EGI, Corp., Eugene, Oregon). All processing and visualisation was accomplished using both the EEGLAB toolbox (v13.1.1) and code composed by the authors running under Matlab 7.12.0. Bandpass filtering between 1 and $50 \mathrm{~Hz}$, was performed using the EEGLAB default basic FIR filter.

We extracted data for the four conditions (pure AO, pure MI, synchronised AO+MI and static $\mathrm{AO}+\mathrm{MI}$ ) at intervals between -1.5 to $13 \mathrm{~s}$ from the onset of each trial (either placing their right forefinger on the post or pressing space bar). We rejected channels if the average amplitude exceeded $4 S D$ from the overall mean. Noisy epochs were rejected based on extremely large fluctuations (i.e., a 1000 microV threshold limit) and improbable activity (5 $S D$ probability threshold, with a 5\% maximum number of trials being rejected per iteration).

In both the familiarisation (phase 3 and 5) and throughout the main experimental procedures we instructed participants not to make any physical movements during Event B (i.e., during the EEG data collection period). On each trial a researcher watched the participants' behaviour, and this was also filmed using a digital video recorder. In order to ensure movement did not contaminate the data during the main 4 s period of interest (see Figure 2, Event B), potentially problematic trials were checked on the video and individual trials in which participants moved were rejected. Whilst we considered this procedure adequate for the present purposes, future studies could incorporate electromyography (EMG) recordings to investigate if the EEG activity is influenced by differing covert (i.e., below movement threshold) muscle activation levels. 
An independent component analysis (ICA) was performed on short epoched data (-1 to 2 seconds) to isolate artefact-related activity from other contributions to the scalp signals (Delorme \& Makeig, 2004). We copied the resulting ICA weights to the main data set that was previously epoched for longer trials. The ICA data was used as a means of removing any trials with artefacts not identified in the previous step, then re-referenced the data to the average of all electrodes. This resulted in an average of 103 maximally independent EEG components (ICs) for each participant (range $=91$ - 115), which were localized in EEGLAB by fitting a single equivalent current dipole to the scalp potential. ICs were only included in the analysis if they were located within the brain and had less than $15 \%$ residual variance $(\mathrm{RV})$. This procedure resulted in an average of 13.3 components $( \pm 4.7$; range $=6-23)$.

A k-means clustering algorithm separated a total of 405 valid ICs into 29 clusters, corresponding to the common neural sources for the participants (Onton \& Makeig, 2006). When clusters contained more than one IC from an individual participant, the IC with the lowest RV was retained in the cluster. We estimated the neural region represented by each dipole cluster by averaging the Talairach coordinates of the dipoles in each cluster. The anatomical regions that align with these topographical locations are presented in Table 2. We localised six clusters that contained a sufficient number of components for analysis (i.e., 10 - 13 ICs; see Table 2). Wavelet coefficients were estimated at 50 equally spaced frequencies from 3 to $128 \mathrm{~Hz}$ for 200 time points from the pre-stimulus baseline onset (i.e., the appearance of the white cross, -1.5 seconds prior to the start of each trial; see Figure 2), to 12 seconds using Gaussian tapered complex Morlet wavelets (Delorme \& Makeig, 2004).

--- Insert Table 2 about here ---

We computed event-related spectral perturbations in power in each frequency by normalizing the power spectral estimate in each frequency bin by the mean power level during the pre-stimulus baseline. We interpret decreases in spectral power from baseline as an increase in event-related desynchronisation (ERD) in cortical activity or arousal, while an increase in spectral power from baseline indicates a resting state of cortical neural activity (i.e., event-related synchronization: ERS).

We calculated mean spectral power for both the mu- $(8-12 \mathrm{~Hz})$ and beta-bands (15 $30 \mathrm{~Hz}$ ) across the 4s period of interest (Event B in Figure 2), within each of the six clusters, for the four experimental conditions. These values were compared individually to prestimulus baseline values using a series of one-sample t-tests (aim 2.1). 
We investigated the effects of the different experimental conditions on activations in primary sensorimotor and parietal regions using a four-factorial ANOVA. This involved the with-subjects factors of experimental condition (pure AO, pure MI, synchronised AO+MI, static $\mathrm{AO}+\mathrm{MI}$ ), hemisphere (left, right), region (motor, parietal), and frequency band (mu / alpha, beta). We then used simple main effect analyses (i.e., one-way ANOVAs across the four conditions) to investigate the results further, in relation to aims $2.2-2.4$. To pursue aim 2.4 further we used a two-factorial ANOVA on the prefrontal cluster. This involved the within-subject factors of frequency band and experimental condition. We also ran a one-way ANOVA to investigate the effects of experimental condition in the alpha-band data in the occipital cluster. Note that there were no significant differences among the four experimental conditions for any of the clusters that we discarded due to insufficient ICs (i.e., < 10).

All statistical analyses were conducted using SPSS Statistics 21 (IBM). Where appropriate, we adjusted these for any violation of the homogeneity of variance assumption using the Greenhouse-Geisser correction. Alpha levels were set to 0.05, and effect sizes were calculated as partial eta squared values $\left(\eta_{p}{ }^{2}\right)$. To reduce type I error rates, we used Fisher's least significant difference (LSD) contrasts in all pairwise comparisons, since four or less conditions were involved in each comparison (Carmer \& Swanson, 1973).

\section{Results}

\subsection{Behavioural data}

Aim 1.1: Imitation bias across experimental conditions

The simple main effect analyses confirmed the imitation bias was present in the pure MI condition, $\left(F(1,13)=41.09 ; p<0.001 ; \eta_{p}{ }^{2}=0.76\right)$, as well as in each of the four compatibility conditions of both synchronised AO+MI (all $p \mathrm{~s} \leq 0.001$; all $\eta_{\mathrm{p}}{ }^{2} \mathrm{~s} \geq 0.67$ ), and static $\mathrm{AO}+\mathrm{MI}\left(\right.$ all $p \mathrm{~s} \leq 0.05 ;$ all $\left.\eta_{p}{ }^{2} \mathrm{~s} \geq 0.36\right)$.

\section{Aim 1.2: Synchronised AO+MI vs. Pure MI}

In the ratio data (\%) the one-sample t-test confirmed that the imitation bias was stronger for synchronised AO+MI (fully-compatible trials only), relative to pure MI, $t(13)=51 ; p<$ 0.001 ; see Figure 3.

--- Insert Figure 3 about here --- 
Aim 1.3: Synchronised $A O+M I$ vs. static $A O+M I$

In the two-factorial ANOVA the main effect of experimental condition was significant, $F(1$, 13) $=28.5, p<0.001, \eta_{p}{ }^{2}=0.69$, indicating that the slow:fast response ratio was significantly stronger for synchronised AO+MI, compared to static AO+MI. Thus, a crucial prerequisite for the EEG analyses was clearly met. The main effect of compatibility was also significant, $F(2.45,31.81)=6.4, p<0.01, \eta_{p}{ }^{2}=0.33$. Exploring this result further within each AO+MI condition revealed that the present data replicated our earlier findings (c.f., Eaves et al., 2014). That is, in synchronised AO+MI the four compatibility conditions were not significantly different from each other, while in static AO+MI there was a clear compatibility effect, $F(2.36,30.26)=5.67, p<0.01, \eta_{p}{ }^{2}=0.30$. Specifically, the imitation bias was stronger for fully-compatible trials compared to both the DA/DP and SA/DP conditions, ps < 0.01. The imitation bias was also numerically (but not significantly) stronger for SA/SP relative to the incompatible DA/SP condition, $p=0.082$.

\subsection{EEG data}

\section{Summary of time-frequency data across full trials}

Figure 4 provides an overview of the EEG data for all conditions and identified clusters. ERD intensity increased during observation of the start cue (green dot) and display of the instructed action (Event A, 1 to 3s). As in the subsequent main Event B, this activity tended to be lateralised to the left hemisphere. As expected, ERD during Event A were particularly strong in the pure MI and the two AO+MI conditions, all of which involved execution of the instructed action with the right hand at the end of each trial (Event C, 7-11s), when stronger, lateralised ERD were present in the motor and posterior parietal clusters. The ERD in the pure $\mathrm{AO}$ condition during Event $\mathrm{C}$ reflected the presentation of the final action picture, as well as the participants' subsequent verbal response. Next we briefly recapitulate the four aims in the EEG analyses.

--- Insert Figure 4 about here ---

First (aim 2.1), we present the analysis of ERD for all four experimental conditions (pure $\mathrm{AO}$, pure $\mathrm{MI}$, synchronised $\mathrm{AO}+\mathrm{MI}$, static $\mathrm{AO}+\mathrm{MI}$ ) relative to the pre-stimulus baseline. We then report the overall results of the four-factorial ANOVA, which investigated the effects of experimental condition on ERD in both the mu- and beta-bands, within primary 
sensorimotor cortex and parietal cortex. Next (aims $2.2-2.4$ ) we describe the findings from the simple main effect analyses run on these data, and we pursue aim 2.4 further in the prefrontal cluster. We then report the one-way ANOVA performed on the occipital cluster.

Since a series of two-way ANOVAs confirmed both the main effect of compatibility and the two-way interaction between experimental condition and compatibility was not significant in the EEG data, the following analyses collapsed across compatibility. All analyses refer to the main Event B (distractor observation and/or motor imagery).

\section{Aim 2.1: Experimental conditions vs. pre-stimulus baseline}

ERD were significantly stronger for the four experimental conditions, relative to baseline in almost all comparisons (i.e., across bands and all six clusters; all $p \mathrm{~s}<0.05$ ). The only two exceptions were both in the right parietal alpha-band, where pure AO and pure MI yielded ERD numerically (but not significantly) stronger than baseline ( $p$ s $=0.07$ and 0.19 , respectively).

Overall ANOVA results: Effects of experimental condition on mu- and beta-ERD in left and right motor and parietal regions

The four-factorial ANOVA revealed a significant main effect of experimental condition, $F(2.4,22.4)=18.1, p<0.001 ; \eta_{p}{ }^{2}=0.67$. Pairwise comparisons identified that the synchronised AO+MI condition produced significantly stronger ERD, compared both to pure $\mathrm{AO}$, and to pure MI ( $p s \leq 0.01$, see also Figures 4 and 5). No reliable differences between the two AO+MI conditions were found $(p>0.05)$. In addition, there was a trend for stronger ERD for pure MI, compared to pure AO $(p=0.06)$. All other main effects (i.e., for hemisphere, region, and band) and interactions were not significant. For example, we only observed a trend for stronger ERD in the left, compared to the right hemisphere across the four clusters analysed, $F(1,9)=3.7, p=0.09 ; \eta_{p}{ }^{2}=0.29$. However, when the analysis was restricted to the motor cortex, a significant main effect of hemisphere was found $F(1,11)=$ $6.95, p=0.023 ; \eta_{p}{ }^{2}=0.39$, corroborating previous studies showing stronger ERD during motor imagery in the hemisphere involved in motor execution of the imagined action (e.g., Neuper et al., 2009; Burianová et al., 2013).

--- Insert Figure 5 about here --- 


\section{Aim 2.2: Synchronised $A O+M I$ vs. (a) pure $A O$ and (b) pure $M I$}

As shown in Table 3, simple main effect analyses revealed that ERD were significantly stronger for synchronised AO+MI, compared to pure AO (aim 2.2a), across bands in the left and right motor, and in the left parietal cluster (all $p$ s $<0.05$ ). A similar but non-significant trend was found in both bands in the right parietal cluster, $p \mathrm{~s} \leq 0.12$.

When compared to pure MI (aim 2.2b), ERD were significantly stronger for synchronised $\mathrm{AO}+\mathrm{MI}$ in the left motor, and bilateral parietal beta-band (all $p \mathrm{~s} \leq 0.01$, see Table 3). A similar but non-significant trend was also found in the left motor mu-band, the parietal alpha-band bilaterally, and the right motor beta-band, all $p \mathrm{~s} \leq 0.16$. Overall then, there was a clear pattern for ERD to be stronger for synchronised AO+MI, relative to the two constituent processes alone (i.e., pure AO and pure MI). This difference was more pronounced when synchronised $\mathrm{AO}+\mathrm{MI}$ was compared to pure $\mathrm{AO}$, rather than to pure MI.

--- Insert Table 3 about here ---

\section{Aim 2.3: Pure MI vs. pure AO}

Direct comparisons indicated that ERD in the right motor mu-band were significantly stronger for pure MI, compared to pure AO, $p<0.01$, see Table 3. In addition, beta-ERD in the right motor cluster were numerically (but not significantly) stronger for pure MI, relative to pure AO, $p=0.16$. Similarly, there was a trend for ERD in the left motor beta-band to be stronger during pure $\mathrm{MI}$, compared to pure $\mathrm{AO}, p=0.12$. All other comparisons were not significant. Overall, these results indicate only a moderate trend for ERD in the primary sensorimotor cortex to be stronger for pure MI, compared to pure AO.

Interestingly, a rapid rebound appears to occur in the left motor cluster prior to movement execution during the MI simulation interval. Since we collapsed epochs of interest and did not analyse the data across time bins, it is impossible to say whether or not the rebound is significantly different compared to the other conditions. Regardless, ERD rebound has been shown to occur after movement in the contralateral hemisphere, and bilaterally after action observation (Avenzini et al., 2012). Since participants were imagining/performing the task exclusively with their right hand, ERD over the right motor cortex was weak compared to the left. Because of this, it is difficult to determine if the rebound effect was bilateral. 


\section{Aim 2.4: Synchronised vs. static AO+MI}

Consistent with the overall ANOVA results, simple main effect analyses found no significant differences between the two AO+MI conditions in both the primary sensorimotor and parietal regions across both bands (all $p \mathrm{~s}>0.05$ ). In contrast, the two-factorial ANOVA on the left rostral prefrontal cluster revealed a significant main effect of condition, $F(3,33)=4.21$, p < $0.05 ; \eta_{p}{ }^{2}=0.27$. See Figures 4 and 6 . Pairwise comparisons showed ERD were significantly stronger for synchronised AO+MI, compared to static AO+MI, in both frequency bands. In addition, synchronised AO+MI produced significantly stronger ERD relative to both pure $\mathrm{AO}$, and pure MI. Both the main effect of band and the interaction between experimental condition and band was not significant in the two-factorial ANOVA. Importantly, the findings for the prefrontal cluster identify a pattern of neural activation that was unique to the synchronised AO+MI condition.

--- Insert Figure 6 about here ---

\section{Occipital activity}

The one-way ANOVA on the alpha-band data in the occipital cluster revealed a significant main effect of experimental condition, $F(2.6,31.7)=7.83, p=0.001 ; \eta_{p}{ }^{2}=0.40$.

Unsurprisingly, pairwise comparisons showed that ERD were numerically (but not significantly) stronger for pure AO, compared to pure MI $(p=0.08)$, where after an initial burst of activity at the onset of event B (purple cross) levels quickly returned to near baseline. Finally, in both $\mathrm{AO}+\mathrm{MI}$ conditions ERD in the occipital cluster were stronger compared to both pure $\mathrm{AO}$ and pure $\mathrm{MI}(p \mathrm{~s}<0.05)$. Involvement of visual cortex during motor imagery has been reported previously (e.g., Burianová et al., 2013; see also Hetu et al., 2013), which most likely indicates an extended network of motor imagery involving visuo-spatial representations for action. Alternatively, while we had certainly instructed a focus on kinesthetic motor imagery, it is possible that participants had also spontaneously engaged in visual imagery of the instructed action, and particularly so in the presence of the distractor movies. 


\section{Discussion}

\subsection{Behavioural results}

First we found that, following pure MI, the cycle time of the participants' imagined action significantly biased the cycle time of their subsequently executed action. In our previous studies no pure MI condition was included, thus this novel finding nicely complements our previous results and adds to the large body of evidence on behavioural effects of MI (see for reviews Munzert et al., 2009; Schuster et al., 2011).

Second, we confirmed our prediction that the imitation bias would be significantly stronger for synchronised AO+MI than for pure MI (aim 1.2). Such a result is tentative evidence against the idea that the motor imagery component of AO+MI might be the only driver of behavioural effects of $\mathrm{AO}+\mathrm{MI}$ procedures. Rather, our result suggests a clear contribution of action observation to the overall effect of synchronised $\mathrm{AO}+\mathrm{MI}$. One possible interpretation is sensu direct visuo-motor priming effects (see Introduction). Note, however, that in the present experimental task pure MI was only preceded, but not accompanied by observation of the to-be-imagined tempo, and so did not involve opportunities for updating the required tempo on-line in the same way as synchronised AO+MI did (see Figure 2, Events A and B). Whilst this lack of visual input is simply a defining (and potentially restricting) feature of pure $\mathrm{MI}$, these considerations also point to a limitation of tasks such as ours in unambiguously demonstrating a net effect of action observation within AO+MI. Moreover, the stronger bias found here for synchronised AO+MI could indeed have resulted from this on-line guidance of MI by the visual input, rather than by separable, direct effects of $\mathrm{AO}$ on motor output. Thus, further evidence for the contribution of $\mathrm{AO}$ during $\mathrm{AO}+\mathrm{MI}$ would be desirable from tasks with dependent measures that reflect the benefits of concurrent visual input for MI to a smaller extent than the present paradigm. In terms of differential practice effects of synchronised AO+MI vs. pure MI, it follows that tasks such as ours, where updating processes during MI are potentially useful, should most clearly bear out the benefits of $\mathrm{AO}+\mathrm{MI}$ procedures over pure $\mathrm{MI}$.

Third, the data for synchronised and static AO+MI replicate our previous results (Eaves et al., 2014) in that the imitation bias was markedly reduced after static, compared to synchronised AO+MI (aim 1.3). This result was a crucial prerequisite for the electrophysiological analyses. It confirms a clear effect of the specific contents of MI during $\mathrm{AO}$, in that conflicting (here: static) $\mathrm{AO}+\mathrm{MI}$ can reduce behavioural effects relative to synchronised AO+MI. Previously (ibid.) we suggested that engaging in motor imagery which conflicts with concurrent $\mathrm{AO}$ might inhibit the default coupling between $\mathrm{AO}$ and motor 
processing (i.e., inhibit automatic imitation). Finally, we also replicated our earlier finding that coordinative $\mathrm{AO}+\mathrm{MI}$, where participants coordinated the observed action with their imagery of a different action type and/or plane or motion (i.e., incongruent synchronised $\mathrm{AO}+\mathrm{MI}$ conditions), produced a similarly strong imitation bias to congruent synchronised AO+MI. Given the wide variety of possible applications of coordinative AO+MI (Vogt et al., 2013), this too is an important and encouraging result. Next we discuss the neurophysiological effects associated with these behavioural findings. Whereas the behavioural data refer to the effects of the different motor simulation states on subsequent execution, the electrophysiological data reflect these simulation states more directly.

\subsection{EEG results}

$\mathrm{Mu}$ - and beta-ERD during $\mathrm{AO}$ and/or MI is a reliable index of premotor and/or motor cortical involvement during covert motor simulation states (see Introduction). Although we did not aim for precise anatomical assignments in the present study, it is likely that these ERD indicate activity in regions of the human action observation network (e.g., Caspers et al., 2010; Naish et al., 2014) and/or the human mirror neuron system, where the latter refers more specifically to an action observation/execution matching system supporting action simulation processes (Rizzolatti \& Sinigaglia, 2010; Kilner \& Lemon, 2013).

Regarding our first aim in the EEG analyses (2.1), we showed that both mu- and betaERD in the left hemisphere were significantly stronger during all four experimental conditions, compared to baseline. These results plainly replicate the solid body of multimodal neuroimaging research indicating the involvement of motor cortical areas in AO (Caspers et al., 2010; Naish et al., 2014; Babiloni et al., 2002) and MI (Hetu et al., 2013; Kraeutner et al., 2014; Llanos et al., 2013).

Regarding our second aim (2.2) we demonstrated that synchronised AO+MI increased ERD in primary sensorimotor cortex, as well as in posterior parietal regions, relative to both our pure $\mathrm{AO}$ and pure MI conditions, using a single within-subjects paradigm. The results for synchronised $\mathrm{AO}+\mathrm{MI}$ vs. pure $\mathrm{AO}$ complement the recent neuroimaging and TMS studies (see Introduction). In our study, the pure AO condition did not involve subsequent motor execution. While this might be perceived as a confound, we argue that $\mathrm{AO}$ conditions involving subsequent execution might actually lead participants to spontaneously engage in AO+MI, which we were seeking to avoid here. For example, in the study by Roosink and Zijdewind (2010) the higher corticospinal excitability during AO in order to imitate, 
compared to that during passive AO, could well be explained in this way. Given the available evidence, the conclusion that (explicit or implicit) synchronised $\mathrm{AO}+\mathrm{MI}$ involves stronger neural activity than pure $\mathrm{AO}$ is then rather safe.

Regarding the contrast between AO+MI and MI (aim 2.2b), the existing evidence is more sparse and less clear-cut. Namely, the sample size was small in Sun et al.'s (2014) preliminary study in post-stroke patients $(n=2)$, while the interpretation was compromised in Taube et al.'s (2015) work by a lack of counterbalancing, and in both Mouthon et al.'s (2015) and Wright et al.'s (2014) studies there was only a trend for corticospinal excitability to be enhanced in $\mathrm{AO}+\mathrm{MI}$ relative to MI. The present data therefore represent the first clear-cut evidence for stronger neural activity during synchronised AO+MI than during pure MI. Together with the related behavioural finding (aim 1.2), these results provide consistent evidence for a dual-simulation account of $\mathrm{AO}+\mathrm{MI}$. That is, we conclude that the activity in motor-related cortical regions during synchronised $\mathrm{AO}+\mathrm{MI}$ reflects the combined activity of the individual neural simulations during AO and MI. Since the neurocognitive processes during AO and MI are likely to overlap at least in part, no straightforward additivity of the ERD signal should be expected.

For completeness, we also contrasted the ERD during pure AO and pure MI directly (aim 2.3). This indicated a trend for stronger activity in sensorimotor cortex, but not in posterior parietal cortex, for pure MI (Figures 4 and 5). This pattern of results is in line with the conclusions from the early meta-analysis by Grezes and Decety (2001), as well as with the more recent results by Filimon et al. (2007; 2014), Szameitat et al. (2012), and Wright et al. (2014). We abstain from interpreting the relatively small differences between neural activity to indicate a possible 'more direct' access of MI to motor processes, compared to AO. Conversely, these results would caution against any generalisations that $\mathrm{AO}$ might be a preferable mode of motor simulation compared to MI for motor learning (e.g., Gatti et al., 2013; Gonzalez-Rosa et al., 2015). Indeed, when any incentive for spontaneous motor imagery in a putative pure AO condition is removed, as in the present study, then pure MI demonstrates at least the same degree of motor cortical involvement as pure, 'passive' AO.

\section{Synchronised $A O+M I$ vs. static $A O+M I$}

The comparison between these two forms of AO+MI was a core objective in the present study (aim 2.4). We found no significant differences between synchronised AO+MI and static $\mathrm{AO}+\mathrm{MI}$ in primary sensorimotor and posterior parietal regions (Figure 5). Both $\mathrm{AO}+\mathrm{MI}$ conditions engaged the visuo-motor system to the same extent, regardless of the 
match between the two component representations, and regardless of the clear behavioural differences between these conditions in subsequent execution. This is not overly surprising, since the AO content was identical in both conditions, and since the MI content was at least similar (i.e., dynamic vs. static imagery of the same action). While one might have expected, e.g., a mutual ERD facilitation in synchronised AO+MI and mutual inhibition in static $\mathrm{AO}+\mathrm{MI}$, our data provide no evidence of this. Note, however, we had no means to assess the individual neural activity of each representation in the AO+MI conditions, thus it is possible that the relative contributions of each were not the same. For example, static MI might have generated stronger ERD than synchronised MI while supressing AO-related ERD. In future, this issue could be explored using more specific methods, such as multi-voxel pattern analysis of fMRI data (e.g., Filimon et al., 2014).

\section{Prefrontal involvement in the alignment of dual-sensorimotor representations}

In contrast to the above null-effects in sensorimotor and posterior parietal regions, synchronised $\mathrm{AO}+\mathrm{MI}$ produced significantly stronger neural activity in the rostral prefrontal cortex, compared to the other three conditions. According to Fuster's (2008) model of frontal lobe function, planning and executing goal-directed behaviour is organised hierarchically, from the most elementary actions in the motor cortex, to the most complex and abstract behaviours in the lateral prefrontal cortex (PFC). An interpretation of the present finding in terms of increased demands on working memory (ibid.) is rather unlikely, however, since a delayed response was required in all four experimental conditions. Furthermore, our finding is unlikely to reflect increased demands from dual-action simulation per se, since static $\mathrm{AO}+\mathrm{MI}$ attenuated the PFC activity. A more viable interpretation might be in terms of the stronger demands for cognitive control in synchronised AO+MI compared to the other three conditions, as discussed next.

In pure AO, attention can be mainly focused on sensory inputs arising from stimuli external to the body (i.e., stimulus-oriented processing). In contrast, pure MI does not rely on external stimuli, but here attention is presumably focussed on an internal, self-generated representation. Our static AO+MI condition is similar to pure MI in this respect: whilst static $\mathrm{AO}+\mathrm{MI}$ involved external and internal 'sources', participants were asked to maintain the 'internal' motor image and not to align this with the rhythmical action shown. Only the synchronised $\mathrm{AO}+\mathrm{MI}$ condition required such alignment between the external and internal sources. 
More generally, when reviewing the spectrum of different AO+MI states, Vogt et al. (2013) suggested that many daily tasks are likely to require attention to be flexibly allocated toward one source of information without excluding information arriving from the other (e.g., mentally rehearsing a sports movement while watching your opponent). As suggested by Burgess et al. $(2005,2007)$, a possible role of rostral PFC is to route attention between these different sources of information without being directly involved in either stimulus-orientated or stimulus-independent processing, or even in any domain-specific processing per se. Instead rostral PFC might act as a routing system (much like a railway switch-point), determining the focus of future processing (see Burgess et al., 2005, 2007). Support for this idea comes from studies of attentional switching between perceptual and internal representations (e.g., Gilbert, et al., 2005; Henseler et al., 2011; Orr \& Banich, 2014) in prospective memory tasks (Benoit et al., 2012; Burgess et al., 2003), self-other social comparisons (Benoit et al., 2010; Moore et al., 2014, see Wagner, et al., 2012), mentalising for both social and non-social construal (Baetens et al., 2014), and also in sustained attention (Coull et al., 1996).

For our study, Burgess et al.'s gateway hypothesis should indeed predict increased neural activity in rostral PFC for synchronised AO+MI only, since only this task required ongoing reallocations of attention between the component $\mathrm{AO}$ and $\mathrm{MI}$ representations. In line with this tentative task analysis, the requirement to align observed and imagined actions produced the strongest imitation bias in the present study, rather than maintaining the representations per se, as in the static $\mathrm{AO}+\mathrm{MI}$ condition. As such, and subject to confirmation by future research, the present finding points to a possible role for the rostral prefrontal cortex in aligning dual-sensorimotor representations.

One recent study (Meirovitch et al., 2015) indicated that violation vs. compliance to the 2/3 power law of observed dot trajectories modulated decreases in alpha power over midline motor areas, and in beta power over frontal areas. Whilst we did not find differential effects between these bands in the two AO+MI conditions, one might still argue that the enhanced frontal beta during synchronised AO+MI could simply be driven by the requirement to engage in two dynamic action simulations concurrently. After all, such a requirement was not present in the other conditions. While we acknowledge this as a possible interpretation, we believe that the previously cited evidence (i.e., Burgess et al., 2005, 2007) strongly suggests that frontal beta-ERD was the result of greater cognitive control exerted during synchronised AO+MI. 


\section{Conclusions}

Participants consistently report that they can engage in AO+MI with relative ease, and it is clear that $\mathrm{AO}+\mathrm{MI}$ procedures have great potential for applications in sensorimotor training and rehabilitation. In theoretical terms, a dual-simulation account provides a straightforward explanation of these AO+MI procedures (Vogt et al., 2013; Eaves et al., 2014), and it is well grounded in parallel models of action specification. In particular, Cisek and Kalaska (2010) have argued that neurophysiological data obtained across many human and animal studies show that multiple sensorimotor representations can be maintained simultaneously prior to movement execution, before a single action is selected for execution on the basis of contextdependent information. The present study provides two lines of evidence which support and specify our dual-simulation account of AO+MI.

First, the neural activity during AO+MI was found to be stronger than that for either pure AO or pure MI. This provides further evidence against the possible notion that AO+MI states might be reducible to one of the two component processes. Whilst there is already sufficient evidence available indicating that neural activity during $\mathrm{AO}+\mathrm{MI}$ is enhanced relative to pure AO (as replicated in the present study), only a few studies have contrasted $\mathrm{AO}+\mathrm{MI}$ and pure MI, with less clear-cut results (see Discussion). It is thus at least conceivable that the $\mathrm{MI}$ component of $\mathrm{AO}+\mathrm{MI}$ might be the main driver of both the behavioural and neurophysiological effects of AO+MI. Indeed, in the only available study to date contrasting behavioural outcomes of AO+MI with MI (Taube et al., 2014), the practice effects of these two conditions did not significantly differ (but see their discussion for caveats). The present study, however, provides two pieces of evidence against this view: In the behavioural data, synchronised $\mathrm{AO}+\mathrm{MI}$ produced an imitation bias almost twice as large as pure MI. Further studies are now needed to confirm or specify this finding in a range of other motor tasks. In addition, the electrophysiological data indeed mirrored the behavioural data in this respect, that is, neural activity in both primary sensorimotor and posterior parietal regions was markedly stronger for AO+MI than for pure MI. These novel findings provide strong and convergent support for the dual-simulation account of AO+MI. Supporting this further, we also found that the ERD intensities during pure AO and pure MI were fairly similar in the present task, with only slightly stronger activity during pure MI overall.

The second contribution of the present study is the novel comparison between two contrasting forms of AO+MI (synchronised and static), which are instances of congruent/coordinative and conflicting AO+MI states, respectively, sensu Vogt et al. (2013). 
While we replicated Eaves et al.'s (2014) behavioural finding that static AO+MI strongly reduced the imitation bias relative to synchronised AO+MI, the ERD intensities were equally strong for both forms of AO+MI. Most likely, the ERD reflected the cumulative 'raw' primary sensorimotor activity, which might not be sensitive to the match or mismatch between the two representations. This can now be studied further with more refined methodology such as multi-voxel pattern analysis of fMRI data.

In contrast to the ERD for sensorimotor and posterior parietal cortex, the ERD for the left rostral prefrontal cluster were significantly stronger during synchronised AO+MI than in the other three conditions, including static AO+MI. We explained this finding with reference to Burgess et al.'s (2007) gateway hypothesis, which maintains "that rostral PFC supports mechanisms that enable us to attend ... either to environmental stimuli, or by contrast, to selfgenerated or maintained representations" (ibid., p. 290). It is indeed very plausible that synchronised $\mathrm{AO}+\mathrm{MI}$ would involve attentional switching between the concurrent external (AO) and internal (MI) representations much more frequently than static AO+MI, pure AO, or pure MI.

Given the ample evidence for low-level entrainment processes, e.g., in inter-manual coordination (Kelso, 1995; 2014), a model where two concurrent sensorimotor representations are kept in check via supervisory (prefrontal cortical) control might appear counterintuitive and unparsimonious and will certainly require considerable further research. At present, it certainly fits our data better than a model where both representations 'resonate' spontaneously with each other. Incidentally, a model of supervisory control involving prefrontal cortex (Shallice, 2004) was also successfully applied to explaining the processes of imitation learning (Buccino et al., 2004; Vogt et al., 2007), and, more recently, observational learning (Higuchi et al., 2012).

More generally, $\mathrm{AO}+\mathrm{MI}$ procedures as studied here can well be seen as a special case of 'joint action' (Sebanz et al., 2006) where different action representations (i.e., own and other) need to be maintained and coordinated. Here, neural mechanisms for low-level entrainment alone would, in most instances, hinder, rather than facilitate goal achievement. In this broader perspective, supervisory control would indeed have a crucial role whenever tasks require multiple action representations, such as in many everyday social interactions.

To conclude, the present results provide substantial evidence supporting a dualsimulation account of $\mathrm{AO}+\mathrm{MI}$ procedures. We have already pointed to the wide potential of $\mathrm{AO}+\mathrm{MI}$ procedures in both motor learning and rehabilitation (e.g., in stroke, brain-injured or neuro-degenerative patients), particularly when physical practice is either restricted or not 
possible due to time or injury constraints. For example, see Sun et al.'s (2014) work for encouraging, preliminary evidence for benefits of $\mathrm{AO}+\mathrm{MI}$ in post-stroke patients. While some previous debates concerned the relative efficacy of pure AO versus pure MI (e.g., Gatti et al., 2013; Gonzalez-Rosa et al., 2015), the evidence provided here would certainly favour combined $\mathrm{AO}+\mathrm{MI}$ procedures over either $\mathrm{AO}$ or $\mathrm{MI}$ alone. This will have to be verified in dedicated studies on the practice effects of AO+MI procedures (see Higuchi et al. 2012) for an early example). From an applied perspective, we can only see one slight snag of AO+MI procedures in comparison to pure $\mathrm{AO}$ or $\mathrm{MI}$, namely that $\mathrm{AO}+\mathrm{MI}$ seems to require additional neurocognitive resources in the sense of supervisory attentional control.

Footnote 1. Note that we derived the two factors 'action type compatibility' and 'plane compatibility' from pooling the data from their four constituent factors (see Figure 1), namely: (1) instructed action type (face- or surface-oriented), (2) instructed action plane (horizontal or vertical), (3) distractor action type (same or different), and (4) distractor action plane (same or different).

\section{Acknowledgements}

This experiment was carried out by D. Eaves as part of his doctoral thesis, under the supervision of S. Vogt. L. Behmer Jr advised on all aspects of EEG recording and analysis and was supported by the National Science Foundation under Grant Number 0900781. We would like to thank both B. Usherwood and S. Slavin (Lancaster) for their kind help with the technical development and setup of the equipment used in this experiment. Special thanks go to E. Geangu (Lancaster) for her helpful guidance on using the EGI system.

\section{References}

1. Avanzini, P., Fabbri-Destro, M., Dalla Volta, R., Daprati, E., Rizzolatti, G., \& Cantalupo, G. (2012). The dynamics of sensorimotor cortical oscillations during the observation of hand movements: an EEG study. PLoS ONE, 7, e37534.

2. Babiloni, C., Babiloni, F., Carducci, F., Cincotti, F., Cocozza, G., Del Percio, C., Moretti, D. V., \& Rossini, P.M. (2002). Human cortical electroencephalography (EEG) rhythms during the observation of simple aimless movements: a high-resolution EEG study. Neuroimage, 17, $559-572$. 
3. Baetens, K., Ma, N., Steen, J. \& Van Overwalle, F. (2014). Involvement of the mentalizing network in social and non-social high construal. Social, Cognitive and Affective Neuroscience, 9, 817-824.

4. Behmer Jr., L.P., \& Jantzen, K.J. (2011). Reading sheet music activates the mirror neuron system of musicians: An EEG study. Clinical Neurophysiology, 122, 1342-1347.

5. Beisteiner, R., Höllinger, P., Lindinger, G., Lang, W., \& Berthoz, A. (1995). Mental representations of movements. Brain potentials associated with imagination of hand movements. Electroencephalography and Clinical Neurophyiology, 96, 183-193.

6. Benoit, R.G., Gilbert, S.J., Frith, C.D., \& Burgess, P.W. (2012). Rostral prefrontal cortex and the focus of attention in prospective memory. Cerebral Cortex, 22, 1876-1886.

7. Benoit, R.G., Gilbert, S.J., Volle, E., \& Burgess, P.W. (2010). When I think about me and simulate you: medial rostral prefrontal cortex and self-referential processes. Neuroimage, 50, 1340-1349.

8. Berends, H.I., Wolkorte, R., Ijzerman, M.J., \& van Putten, M.J.A.M. (2013). Differential cortical activation during observation and observation-and-imagination. Experimental Brain Research, 229, 337-345.

9. Buccino, G., Vogt, S., Ritzl, A., Fink, G.R., Zilles, K., Freund, H-J., \& Rizzolatti, G. (2004). Neural circuits underlying imitation learning of hand actions: An event-related fMRI study. Neuron, 42, 323-334.

10. Burgess, P.W., Scott, S.K., \& Frith, C.D. (2003). The role of the rostral frontal cortex (area 10) in prospective memory: a lateral versus medial dissociation. Neuropsychologia, 41, 906918.

11. Burgess, P.W., Simons, J.S., Dumontheil, I., \& Gilbert, S.J. (2005). The gateway hypothesis of rostral prefrontal cortex (area 10) function. In J. Duncan, L. Philips, \& P. Mcleod (Eds.), Measuring the Mind: Speed Control and Age (pp. 215-246). London, England: Oxford University Press.

12. Burgess, P.W., Dumontheil, I., \& Gilbert, S.J. (2007). The gateway hypothesis of rostral prefrontal cortex (area 10) function. Trends in Cognitive Science, 11, 290-298.

13. Burianová, H., Marstaller, L., Sowman, P., Tesan, G., Rich, A.N., Williams, M., Savage, G., \& Johnson, B.W. (2013). Multimodal functional imaging of motor imagery using a novel paradigm. Neuroimage, 71, 50-58.

14. Calmels, C., Holmes, P., Jarry, G., Lévèque, J. M., Hars, M., \& Stam, C. J. (2006). Cortical activity prior to, and during, observation and execution of sequential finger movements. Brain Topography, 19, 77-88. 
15. Carmer, S.G., \& Swanson M.R. (1973). An evaluation of ten pairwise multiple comparison procedures by Monte Carlo methods. Journal of American Statistical Association, 68, 66-74.

16. Caspers, S., Zilles, K., Laird, A.R., \& Eickhoff, S.B. (2010). ALE meta-analysis of action observation and imitation in the human brain. Neuroimage, 50, 1148-1167.

17. Cevallos, C., Zarka, D., Hoellinger, T., Leroy, A., Dan, B., \& Cheron, G. (2015). Oscillations in the human brain during walking execution, imagination and observation. Neuropsychologia, 79, 223-232

18. Cisek, P., \& Kalaska, J. F. (2010). Neural mechanisms for interacting with a world full of action choices. Annual Reviews of Neuroscience, 33, 269-298.

19. Coull, J.T., Frith, C.D., Frackowiak, R.S.J., \& Grasby, P.M. (1996). A fronto-parietal network for rapid visual information processing: a PET study of sustained attention and working memory. Neuropsychologia, 34, 1085-1095.

20. Cochin, S., Barthelemy, C., Lejeune, B., Roux, S., \& Martineau, J. (1998). Perception of motion and qEEG activity in human adults. Electroencephalography and Clinical Neurophysiology, 107, 287-295.

21. Cochin, S., Barthelemy, C., Roux, S., and Martineau, J. (1999). Observation and execution of movement: similarities demonstrated by quantified electroencephalography. European Journal of Neuroscience, 11, 1839-1842.

22. Delorme, A., \& Makeig, S. (2004). EEGLab: An open source toolbox for analysis of singletrial EEG dynamics. Journal of Neuroscience Methods, 134, 9-21.

23. Eaves, D..L., Haythornthwaite, L., \& Vogt, S. (2014). Motor imagery during action observation modulates automatic imitation effects in rhythmical actions. Frontiers in Human Neuroscience, 8 , article 28.

24. Eaves, D.L., Turgeon, M., \& Vogt, S. (2012). Automatic imitation in rhythmical actions: Kinematic fidelity and the effects of compatibility, delay and visual monitoring. PLoS ONE, 7, e46728.

25. Filimon F., Nelson J.D., Hagler D.J., Sereno M.I. (2007). Human cortical representations for reaching: mirror neurons for execution, observation, and imagery. Neuroimage. 37, 13151328.

26. Filimon, F., Rieth, C. A., Sereno, M.I., \& Cottrell, G.W. (2015). Observed, executed, and imagined action representations can be decoded from ventral and dorsal areas. Cerebral Cortex, 25, 3144-3158.

27. Fuster, J.M. (2008). The Prefrontal Cortex. London, UK: Elsevier. 
28. Gatti R., Tettamanti A., Gough P.M., Riboldi E., Marinoni L., Buccino G. (2013). Action observation versus motor imagery in learning a complex motor task: a short review of literature and a kinematics study. Neuroscience Letters, 540, 37-42.

29. Gilbert, S.J., Frith, C.D., \& Burgess, P.W. (2005). Involvement of rostral prefrontal cortex in selection between stimulus-oriented and stimulus-independent thought. European Journal of Neuroscience, 21, 1423-1431.

30. Gonzalez-Rosa, J. J., Natali, F., Tettamanti, A., Cursi, M., Velikova, S., Comi, G., et al., \& Leocani, L. (2015). Action observation and motor imagery in performance of complex movements: Evidence from EEG and kinematics analysis. Behavioural Brain Research. 281, 290-300.

31. Grezes, J., \& Decety, J. (2001). Functional anatomy of execution, mental simulation, observation, and verb generation of actions: a meta-analysis. Human Brain Mapping, 12, 119.

32. Henseler, I., Krüger, S., Dechnet, P., \& Gruber, O. (2011). A gateway system in rostral PFC? Evidence from biasing attention to perceptual information and internal representations. Neuroimage, 56, 1666-1676.

33. Hétu, S., Grégoire, M., Saimpont, A., Coll, M.P., Eugène, F., Michon, P.E., \& Jackson, P.L. (2013). The neural network of motor imagery: an ALE meta-analysis. Neuroscience \& Biobehavioral Reviews, 37, 930-949.

34. Heyes, C. (2011). Automatic imitation. Psychological Bulletin, 137, 463-483.

35. Higuchi S., Holle H., Roberts N., Eickhoff S. B., Vogt S. (2012). Imitation and observational learning of hand actions: prefrontal involvement and connectivity. Neuroimage, 59, 16681683.

36. Holmes, P.S., \& Collins, D.J. (2001). The PETTLEP approach to motor imagery: A functional equivalence model for sport psychologists. Journal of Applied Sport Psychology, $13,60-83$.

37. Jeannerod, M. (1994). The representing brain: neural correlates of motor intention and imagery. Behavioural Brain Science, 17, 187-202.

38. Jeannerod, M. (2001). Neural simulation of action: a unifying mechanism for motor cognition. Neuroimage, 14, 103-109.

39. Jeannerod, M. (2006). Motor cognition. Oxford: Oxford University Press.

40. Kelso, J.A.S. (1995). Dynamic patterns: The self-organization of brain and behaviour. MIT press, Cambridge, M.A. 
41. Kelso, J.A.S. (2014) The dynamic brain in action: Coordinative structures, criticality, and coordination dynamics. In D. Plenz and E. Niebur (Eds), Criticality in neural systems. Wiley: Weinheim, Germany.

42. Kilner, J.M., \& Lemon, R.N. (2013). What we know currently about mirror neurons. Current Biology, 23, 1057-1062.

43. Koski, L., Iacoboni, M., Dubeau, M.C., Woods, R.P., \& Mazziotta, J.C. (2003). Modulation of cortical activity during different imitative behaviors. Journal of Neurophysiology, 89, 460471.

44. Kraeutner, S., Gionfriddo, A., Bardouille, T., \& Boe, S. (2014). Motor imagery-based brain activity parallels that of motor execution: Evidence from magnetic source imaging of cortical oscillations. Brain Research, 1588, 81-91.

45. Lang, W., Cheyne, D., Höllinger, P., Gerschlager, W., Lindinger, G. (1996). Electric and magnetic fields of the brain accompanying internal simulation of movement. Cognitive Brain Research, 3, 125-129.

46. Llanos, C., Rodriguez, M., Rodriguez-Sabate, C., Morales, I., \& Sabate, M. (2013). Murhythm changes during the planning of motor and motor imagery actions. Neuropsychologia, 51, 1019-1026.

47. Macuga, K.L., \& Frey, S.H. (2012). Neural representations involved in observed, imagined, and imitated actions are dissociable and hierarchically organized. Neuroimage, 59, 27982807.

48. Meirovitch, Y., Harris, H., Dayan, E., Arieli, A., \& Flash, T. (2015). Alpha and Beta Band Event-Related Desynchronization Reflects Kinematic Regularities. The Journal of Neuroscience, 35, 1627-1637.

49. Moore, W.E., Merchant, J.S., Kahn, L.E., \& Pfeifer, J.H. (2014). 'Like me?': Ventromedial prefrontal cortex is sensitive to both personal relevance and self-similarity during social comparisons. Social, Cognitive and Affective Neuroscience, 9, 421-426.

50. Mouthon, A., Ruffieux, J., Wälchli, M., Keller, M., \& Taube, W. (2015). Task-dependent changes of corticospinal excitability during observation and motor imagery of balance tasks. Neuroscience, 303, 535-543.

51. Munzert, J., Lorey, B., \& Zentgraf, K. (2009). Cognitive motor processes: the role of motor imagery in the study of motor representations. Brain Research Reviews, 60, 306-326.

52. Muthukumaraswamy, S.D., Johnson, B.W., \& McNair, N.A. (2004). Mu rhythm modulation during observation of an object-directed grasp. Cognitive Brain Research, 19, 195-201. 
53. Naish, K.R., Houston-Price, C., Bremner, A.J., \& Holmes, N.P. (2014). Effects of action observation on corticospinal excitability: Muscle specificity, direction, and timing of the mirror response. Neuropsychologia, 64, 331-348

54. Nedelko, V., Hassa, T., Hamzei, F., Schoenfeld, M.A., \& Dettmers, C. (2012). Action imagery combined with action observation activates more corticomotor regions than action observation alone. Journal of Neurologic Physical Therapy, 36, 182-188.

55. Neuper, C., Scherer, R., Wriessnegger, S., \& Pfurtscheller, G. (2009). Motor imagery and action observation: Modulation of sensorimotor brain rhythms during mental control of a brain-computer interface. Clinical Neurophysiology, 120, 239-247.

56. Neuper, C., Wörtz, M., \& Pfurtscheller, G. (2006). ERD/ERS patterns reflecting sensorimotor activation and deactivation. Progress in Brain Research, 159, 211-222.

57. Oldfield, R.C. (1971). The assessment and analysis of handedness: The Edinburgh Inventory. Neuropsychologia, 9, 97-113.

58. Onton, J., \& Makeig, S. (2006). Information-based modelling of event-related brain dynamics, Progress in Brain Research, 9, 99-120.

59. Orr, J.M., \& Banich, M.T. (2014). The neural mechanisms underlying internally and externally guided task selection. Neuroimage, 84, 191-205.

60. Pfurtscheller, G., Brunner, C., Schlögl, A., \& Da Silva, F. L. (2006). Mu rhythm (de) synchronization and EEG single-trial classification of different motor imagery tasks. Neuroimage. 31, 153-159.

61. Pfurtscheller, G., \& Lopes da Silva, F.H. (1999). Event-related EEG/MEG synchronization and desynchronisation: basic principles. Clinical Neurophysiology, 110, 1842-1857.

62. Pfurtscheller, G., \& Neuper, C. (1997). Motor imagery activates primary sensorimotor area in humans. Neuroscience Letters. 239, 65-68.

63. Pfurtscheller, G., Stancaâk Jr A, Neuper C. (1996). Event-related synchronization (ERS) in the alpha band - an electrophysiological correlate of cortical idling: a review. International Journal of Psychophysiology, 24, 39-46.

64. Pineda, J.A. (2005). The functional significance of mu rhythms: Translating "seeing" and "hearing" into "doing”. Brain Research Reviews, 50, 57-68.

65. Ramsey, R., Cumming, J., Eastough, D., \& Edwards, M.G. (2010). Incongruent imagery interferes with action initiation. Brain and Cognition, 74, 249-254.

66. Rizzolatti, G., \& Sinigaglia, C. (2010). The functional role of the parieto-frontal mirror circuit: interpretations and misinterpretations. Nature Reviews Neuroscience, 11, 264-274. 
67. Roosink, M., \& Zijdewind, I. (2010). Corticospinal excitability during observation and imagery of simple and complex hand tasks: implications for motor rehabilitation. Behavioural Brain Research, 213, 35-41.

68. Schmidt, R.C., Richardson, M.J., Arsenault, C., \& Galantucci, B. (2007). Unintentional entrainment to an environmental rhythm: effect of eye tracking. Journal of Experimental Psychology: Human Perception and Performance, 33, 860-870.

69. Schuster, C., Hilfiker, R., Amft, O., Scheidhauer, A., Andrews, B., Butler, J., Kischa, U., \& Ettlin, T. (2011). Best practice for motor imagery: a systematic literature review on motor imagery training elements in five different disciplines. BMC Medicine, 9, 75.

70. Sebanz, N., Bekkering, H., \& Knoblich, G. (2006). Joint action: Bodies and minds moving together. Trends in Cognitive Sciences, 10, 70-76.

71. Shallice, T. (2004). The fractionation of supervisory control. In Gazzaniga M. S. (Ed), The Cognitive Neurosciences. 3rd ed. MIT Press, Cambridge, MA.

72. Szameitat, A.J., Shen, S., Conforto, A., \& Sterr, A. (2012). Cortical activation during executed, imagined, observed, and passive wrist movements in healthy volunteers and stroke patients. Neuroimage, 62, 266-280.

73. Sun, Y., Yin, L., Meng, M., Ma, Y., Luo, Z., \& Bao, F. S. (2014). Facilitating motor imagery practice with synchronous action observation for stroke patients. In Healthcare Innovation Conference (HIC), IEEE, 231-235.

74. Taube, W., Lorch, M., Zeiter, S., \& Keller, M. (2014). Non-physical practice improves task performance in an unstable, perturbed environment: motor imagery and observational balance training. Frontiers in Human Neuroscience, article 972.

75. Taube, W., Mouthon, M., Leukel, C., Hoogewoud, H.-M., Annoni, J.-M., and Keller, M. (2015). Brain activity during observation and motor imagery of different balance tasks: An fMRI study. Cortex, 64, 102-114.

76. Villiger, M., Estévez, N., Hepp-Reymond, M-C., Kiper, D., Kollias, S.S., Eng, K., \& HotzBoendermaker, S. (2013). Enhanced activation of motor execution networks using action observation combined with imagination of lower limb movements. PLOS ONE, 8, e72403.

77. Vogt S. (1996). Imagery and perception-action mediation in imitative actions. Cognitive Brain Research. 3, 79-86.

78. Vogt, S., \& Thomaschke, R. (2007). From visuo-motor interactions to imitation learning: behavioural and brain imaging studies. Journal of Sports Science, 25, 497-517.

79. Vogt, S., Di Rienzo, F., Collet, C., Collins, A., \& Guillot, A. (2013). Multiple roles of motor imagery during action observation. Frontiers in Human Neuroscience, 7, article 807. 
80. Williams, S.E., Cumming, J., Ntoumanis, N., Nordin-Bates, S.M., Ramsey, R., \& Hall, C. (2012). Further validation and development of the Movement Imagery Questionnaire. Journal of Sport \& Exercise Psychology, 34, 621-646.

81. Wagner, D. D., Haxby, J. V., \& Heatherton, T. F. (2012). The representation of self and person knowledge in the medial prefrontal cortex. Wiley Interdisciplinary Reviews: Cognitive Science, 3, 451-470.

82. Wang, T. Deng, J., \& He, B. (2004). Classifying EEG-based motor imagery tasks by means of time-frequency synthesized spatial patterns. Clinical Neurophysiology, 115, 2744-2753.

83. Wright, D.J., Williams, J., \& Holmes, P.S. (2014). Combined action observation and imagery facilitates corticospinal excitability. Frontiers in Human Neuroscience, 8, article 951. 


\section{Figure Legends}

Figure 1. Instructed action stimuli with the factors orientation and dominant plane of motion for the two instructed actions: face washing and painting.

Figure 2. Sequence of events in the four experimental conditions: pure AO, pure MI, synchronised AO+MI, and static AO+MI. Event B was the main period of interest for the EEG analysis, whilst we recorded kinematics during Event $\mathrm{C}$ (except for pure AO). When participants placed their right forefinger in the start location a white cross appeared (prestimulus baseline), followed by a green circle ('get ready'). For pure $A O$ a picture of the 'instructed action' was displayed in Event A (see Methods), and was followed by a movie of the same action being pantomimed (Event B: 'distractor action'). A short pause preceded the final action picture (Event C), when participants verbally reported the match between the actions shown in Events B and C. For pure MI participants observed a short movie of the instructed action (Event A). They then fixated on the purple cross (Event B) while they imagined performing the instructed action with their right hand at the pace shown in the preceding movie. They then executed this action with their right hand at their own preferred pace (Event $\mathrm{C}$ ). For both $A O+M I$ conditions a picture of the instructed action (Event $\mathrm{A}$ ) was followed by a movie showing either the same or a different action ('distractor action') being pantomimed (Event B). For synchronised $A O+M I$ participants imagined performing the instructed action and synchronised this with the observed distractor action. For static $A O+M I$ participants imagined performing a static posture of the instructed action while observing the distractor action. In both $\mathrm{AO}+\mathrm{MI}$ conditions participants then executed the instructed action at their own pace (Event C), as they did in pure MI.

Figure 3. Mean cycle time ratios (\%; with standard error of the mean) for three factors involved in the behavioural data analysis: experimental condition, action type compatibility and plane compatibility. The cycle time ratio in the distractor actions was $150 \%$. Key: AO = action observation; $\mathrm{MI}=$ motor imagery.

Figure 4. Time-frequency plots per cluster. Mean time-frequency data for the four experimental conditions (pure $\mathrm{AO}$, pure $\mathrm{MI}$, synchronised $\mathrm{AO}+\mathrm{MI}$, static $\mathrm{AO}+\mathrm{MI}$ ) in both the mu / alpha $(8-12 \mathrm{~Hz})$ and beta bands $(15-30 \mathrm{~Hz})$ for the six source-localised clusters (see Table 3). The interval between 3 and 7s corresponds to Event B in Figure 2 (distractor 
observation and/or motor imagery). The topographical regions aligned with the following anatomical regions: Left motor strip (BA 4), parietal lobe (BA 39), and rostral prefrontal cortex (BA 9 and BA 10); Right motor strip (BA 6), parietal lobe (BA 39), and occipital cortex (BA 19). Source localisation is displayed on the right hand side using a standard BESA brain model, wherein (a) is displayed in the horizontal plane (Talairach coordinates $47,-13,12 ; 12 \mathrm{ICs})$, (b) is in the coronal plane $(-41,-54,6 ; 10 \mathrm{ICs}),(\mathrm{c})$ and (d) are in the horizontal plane (-23, 43, 30; 12 ICs, and 64, -2, 20; 13 ICs, respectively), and (e) and (f) are in the sagittal plane $(-51,-55,8$; 10 ICs and, -13, -61, 5; BA 19; 13 ICs, respectively). Key: Blue $=$ location of individual ICs in each cluster; red = mean cluster location .

Figure 5. Mean event-related power per cluster. Mean spectral power for each of the six localised clusters during Event B (distractor observation and/or motor imagery, see Fig. 2), shown separately for each experimental condition and frequency band (mu / alpha $=8-12$ $\mathrm{Hz}$, and beta $=15-30 \mathrm{~Hz}$ ). The error bars show standard error of the mean.

Figure 6. Topographical plot for left prefrontal cluster. Mean distribution of activity for the cluster of dipoles located in the left rostral prefrontal cortex. The mean topographical location corresponded with Brodmann's area 9 (BA 9; superior Frontal gyrus), while the locations of the individual dipoles were distributed across BA 9 and BA 10. 
Distractor speed

\begin{tabular}{lcc}
\cline { 2 - 3 } Parameters & Slow & Fast \\
\hline Beats per min & 60 & 90 \\
Cycle times (ms) & 1000 & 667 \\
Total cycles in 2 s & 2 & 3 \\
Total cycles in 4 s & 4 & 6 \\
Slow:fast ratio (\%) & & 150 \\
\hline
\end{tabular}

Table 1. Distractor stimuli specifications. 
Talairach coordinates

\begin{tabular}{|c|c|c|c|c|c|c|}
\hline \multirow[b]{2}{*}{$\begin{array}{l}\text { Hemi- } \\
\text { sphere }\end{array}$} & \multirow[b]{2}{*}{ Anatomical region } & \multirow[b]{2}{*}{$\begin{array}{l}\text { Brodmann's } \\
\text { area }\end{array}$} & & \multirow[b]{2}{*}{$\begin{array}{c}\text { No. of } \\
\text { ICs }\end{array}$} \\
\hline & & & $x$ & $y$ & $z$ & \\
\hline \multirow{3}{*}{ Left } & Primary motor cortex & BA 4 & -47 & -13 & 38 & 12 \\
\hline & Caudal inferior parietal lobe & BA 39 & -41 & -54 & 6 & 10 \\
\hline & Rostral prefrontal cortex & BA 9 and BA10 & -23 & 43 & 30 & 12 \\
\hline \multirow{3}{*}{ Right } & Primary motor cortex & BA 6 & 64 & -2 & 20 & 13 \\
\hline & Caudal inferior parietal lobe & BA 39 & -51 & -55 & 8 & 10 \\
\hline & Occipital lobe & BA 19 & -13 & -61 & 5 & 13 \\
\hline
\end{tabular}

Table 2. Estimated anatomical regions for the dipoles involved in each of the six clusters. 


\section{Left hemisphere Right hemisphere}

\begin{tabular}{ccccccc} 
Aim & Planned comparison & Band & Motor & Parietal & Motor & Parietal \\
\hline 2.2a & Sync. AO+MI vs. pure AO & $\mu / \alpha$ & $* *$ & $*$ & $*$ & $p=0.12$ \\
& & $\beta$ & $* * *$ & $* *$ & $* *$ & $p=0.11$ \\
$2.2 \mathrm{~b}$ & Sync. AO+MI vs. pure MI & $\mu / \alpha$ & $p=0.15$ & $p=0.16$ & & $p=0.06$ \\
& & $\beta$ & $* *$ & $* *$ & $p=0.06$ & $* * *$ \\
2.3 & Pure MI vs. pure AO & $\mu / \alpha$ & & & $* *$ & \\
& & $\beta$ & $p=0.12$ & & $p=0.16$ & \\
2.4 & Sync. AO+MI vs. static AO+MI & $\mu / \alpha$ & & & & \\
\hline
\end{tabular}

Table 3. Summary of EEG statistical findings for the four clusters source-localised to primary sensorimotor regions. Main effects and planned comparisons performed in both bands within each of the four clusters $(\mu / \alpha=8-12 \mathrm{~Hz} ; \beta=15-30 \mathrm{~Hz})$. Key for significance values: $*<0.05 ; * *<0.01 ; * * *<0.001$; if cell is blank $\mathrm{p}>0.05$. 


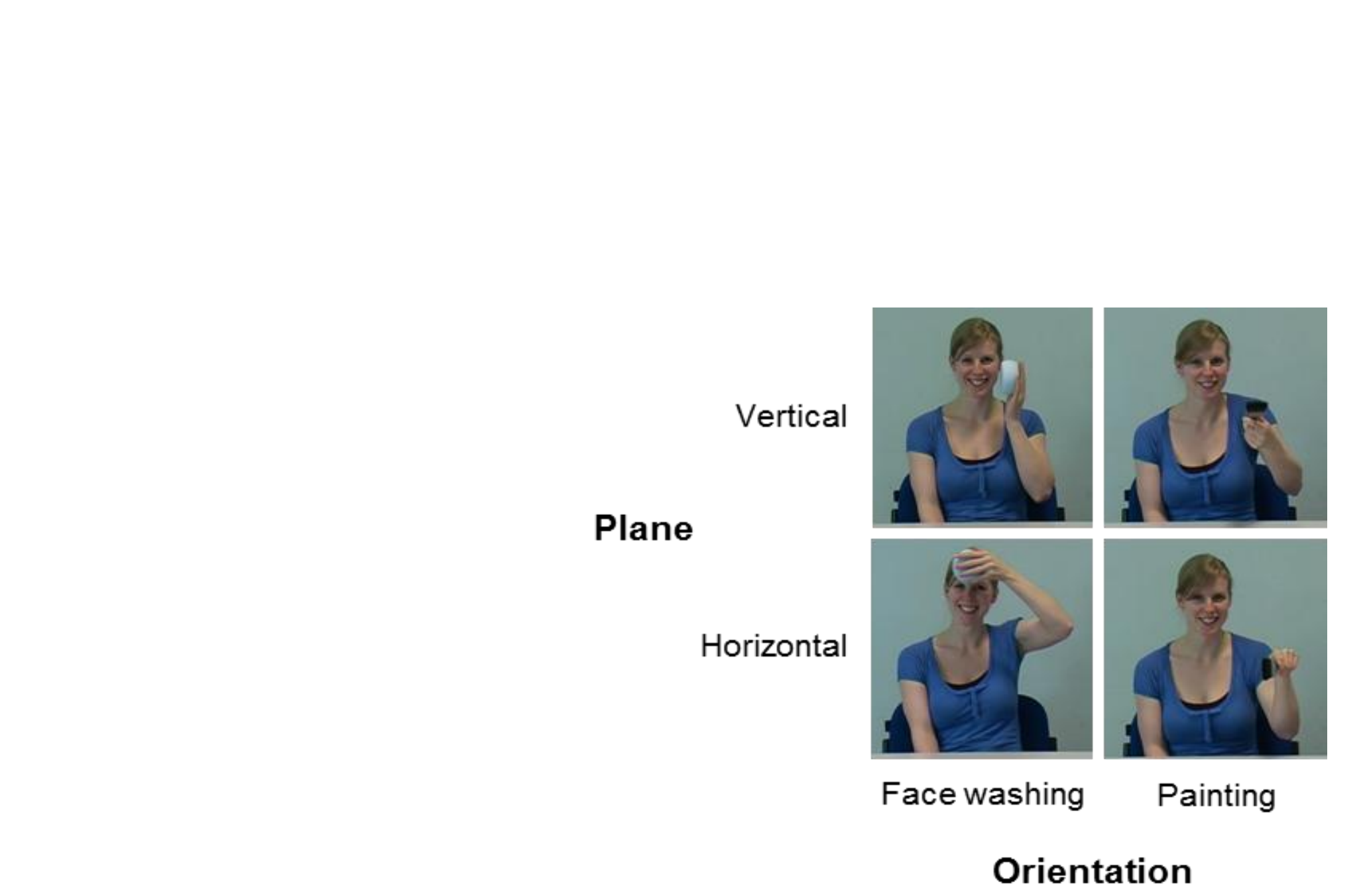

Orientation

Face washing

ainting

Horizontal



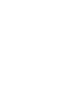

.

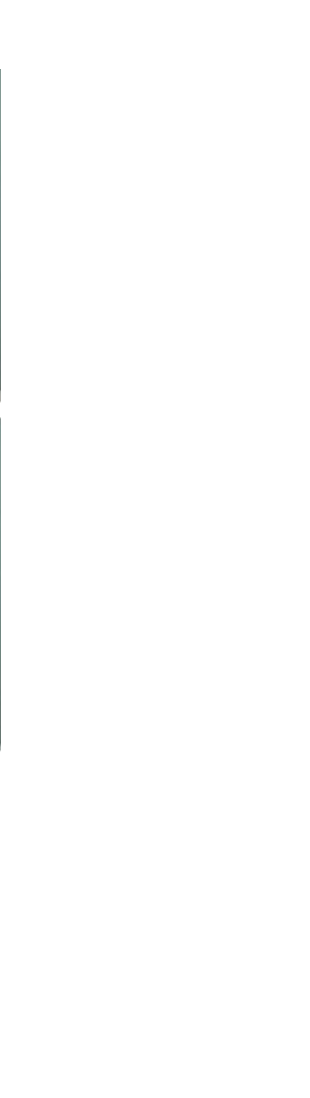






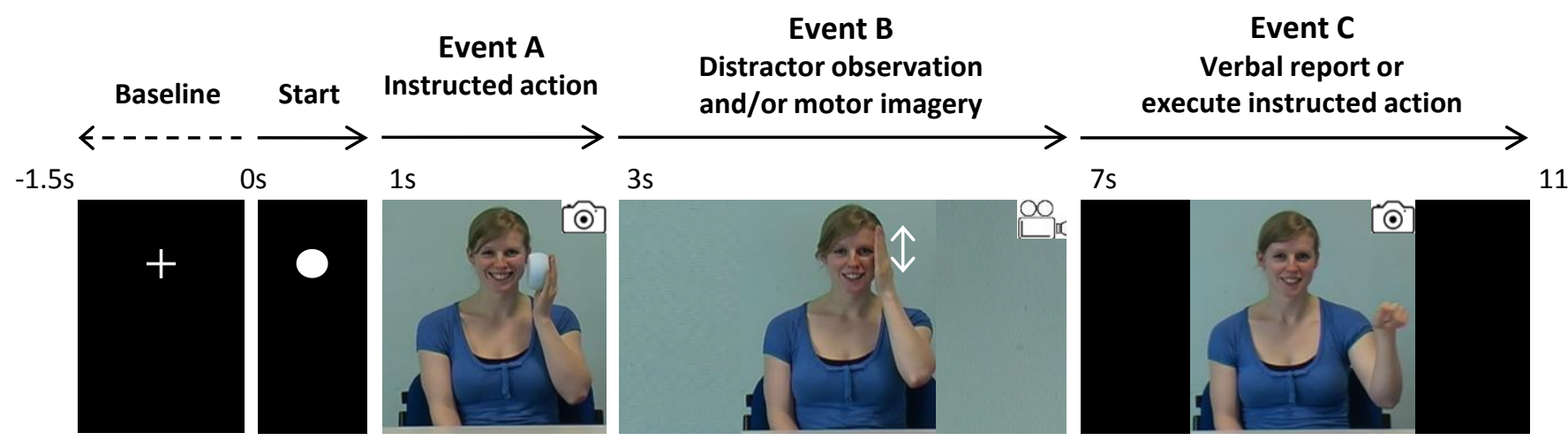

Pure Action Observation (AO): "Verbally report correspondence between actions in Event B and C"

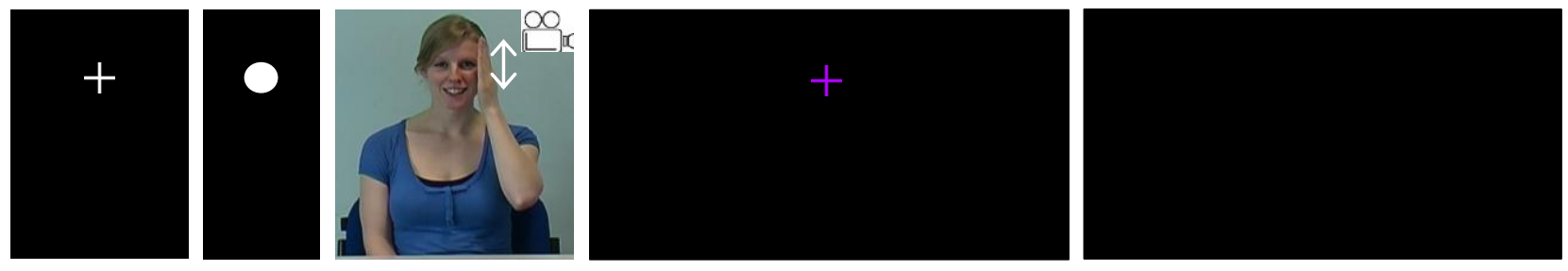

Pure Motor Imagery (MI): “Imagine instructed action during Event B, then execute during Event C"


AO+MI Conditions: "Imagine instructed action (synchronised or static) during Event B, then execute during Event C" 


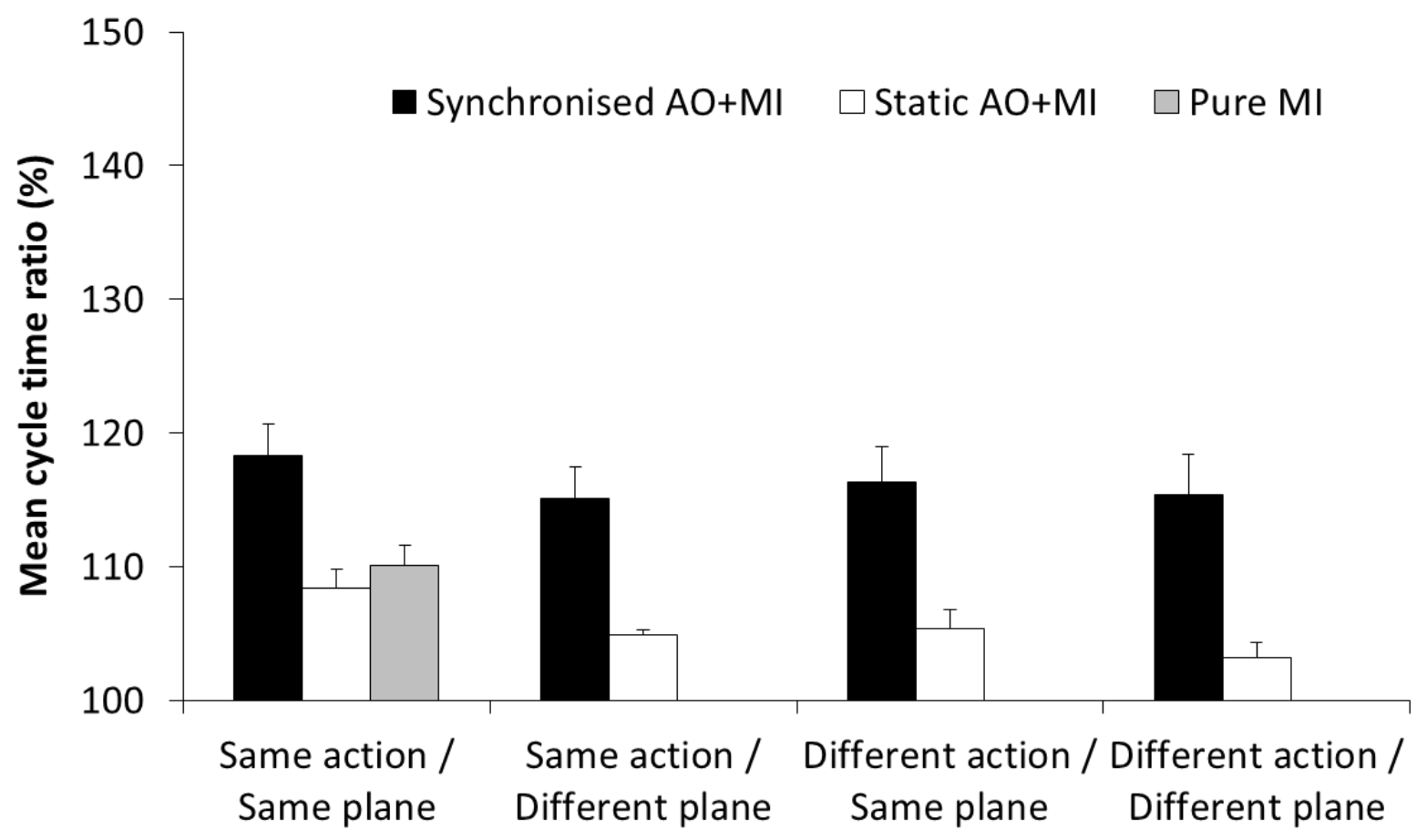

Compatibility condition 
Figure 4
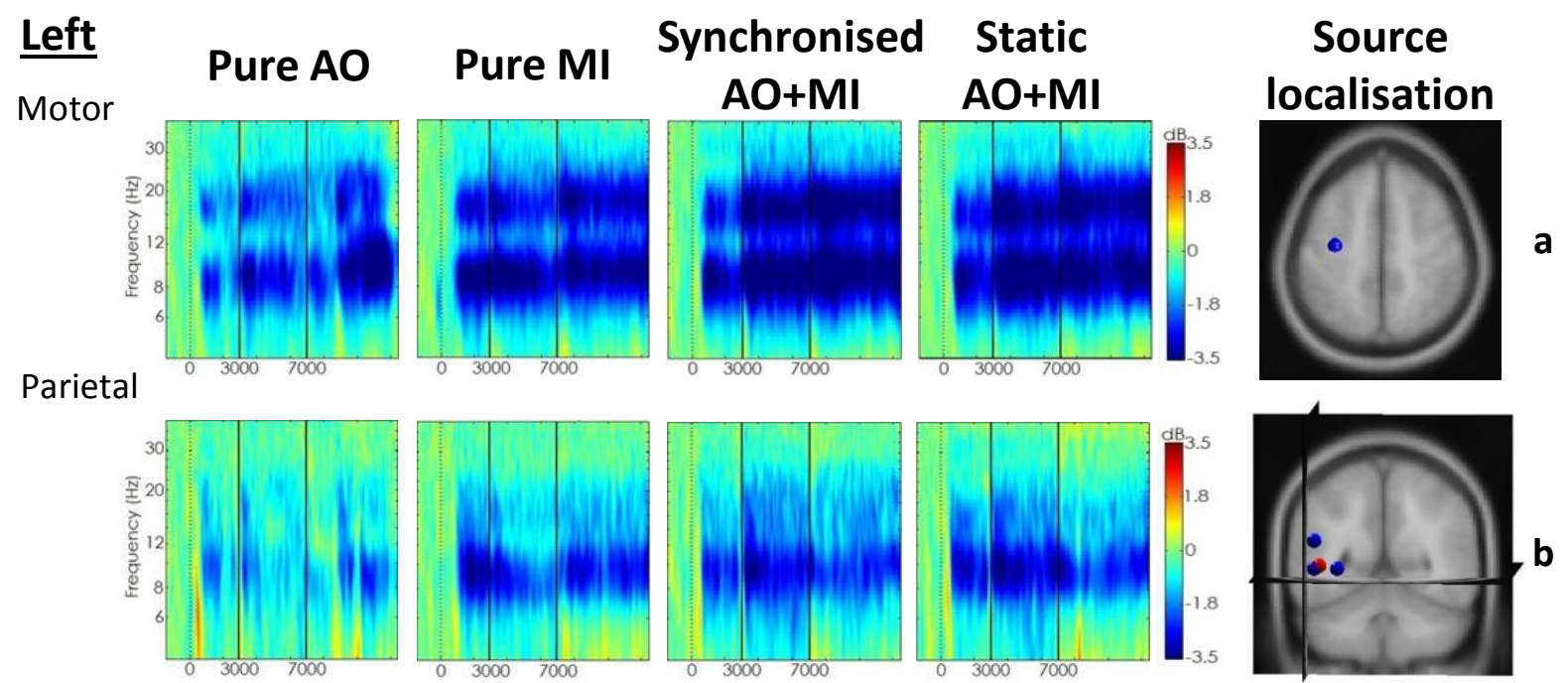

Pre-frontal
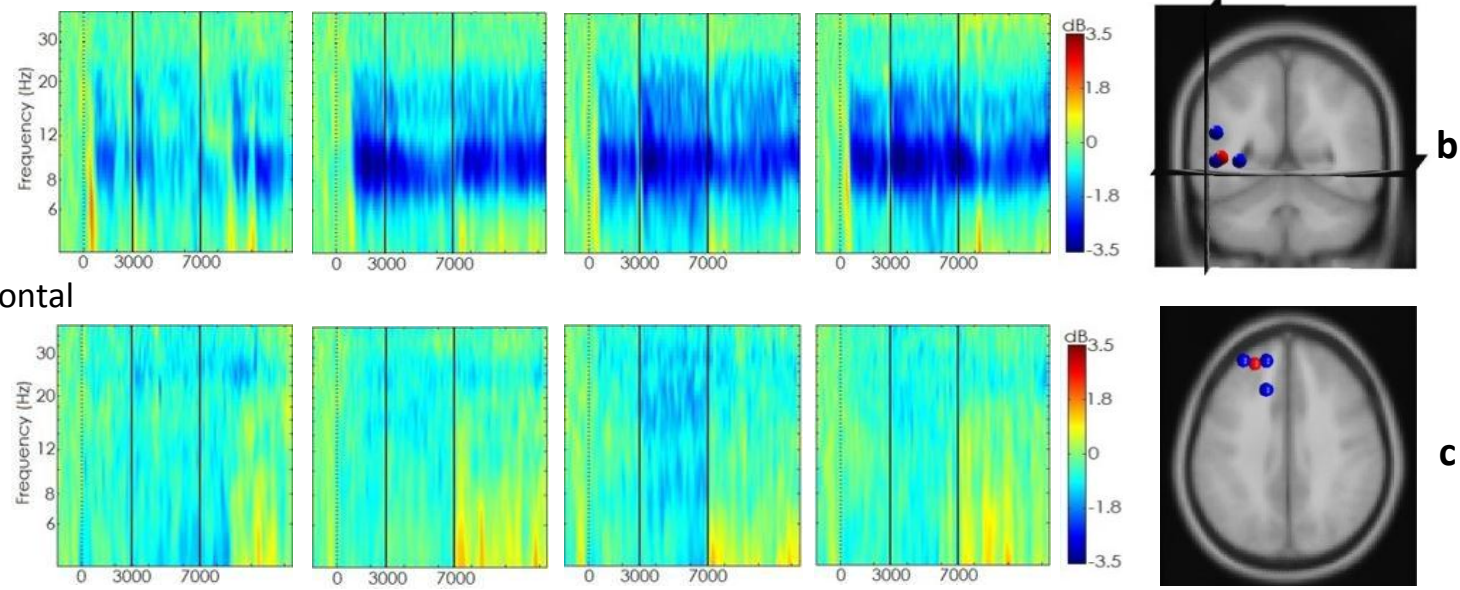

\section{Right}

Motor
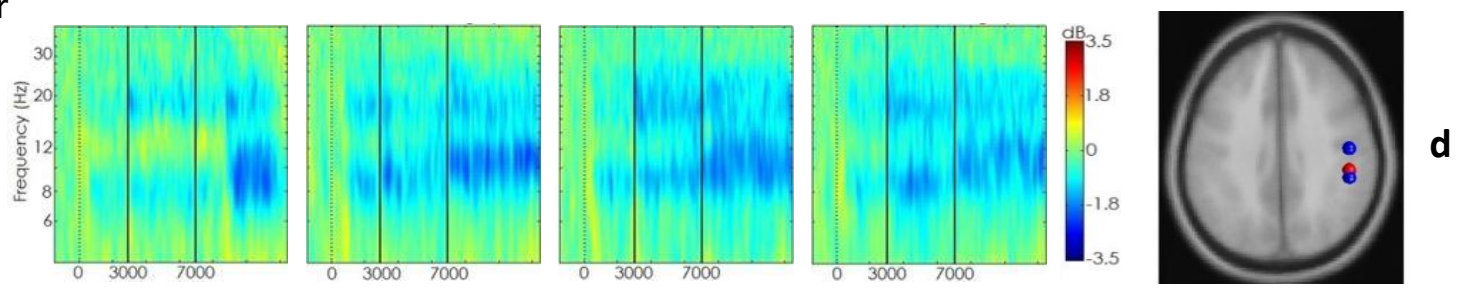

Parietal
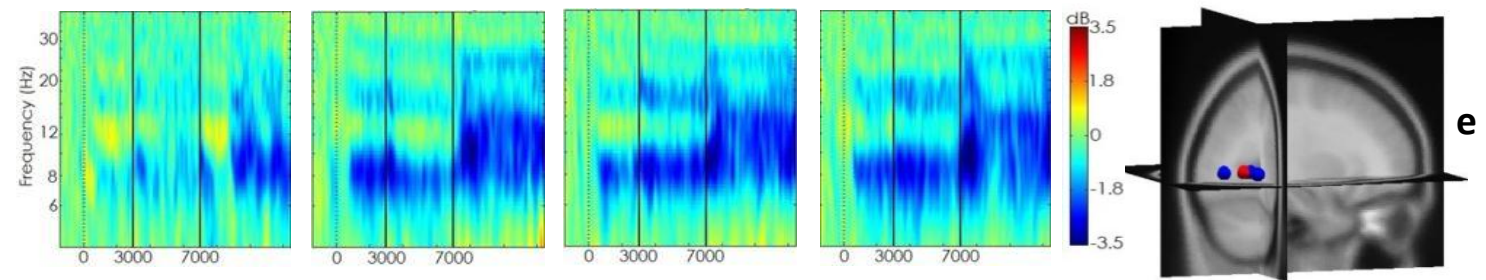

Occipital
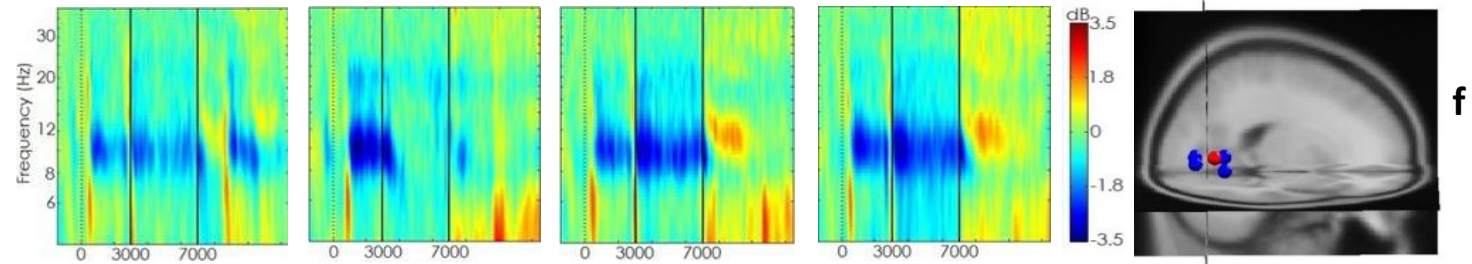

Time (ms) 

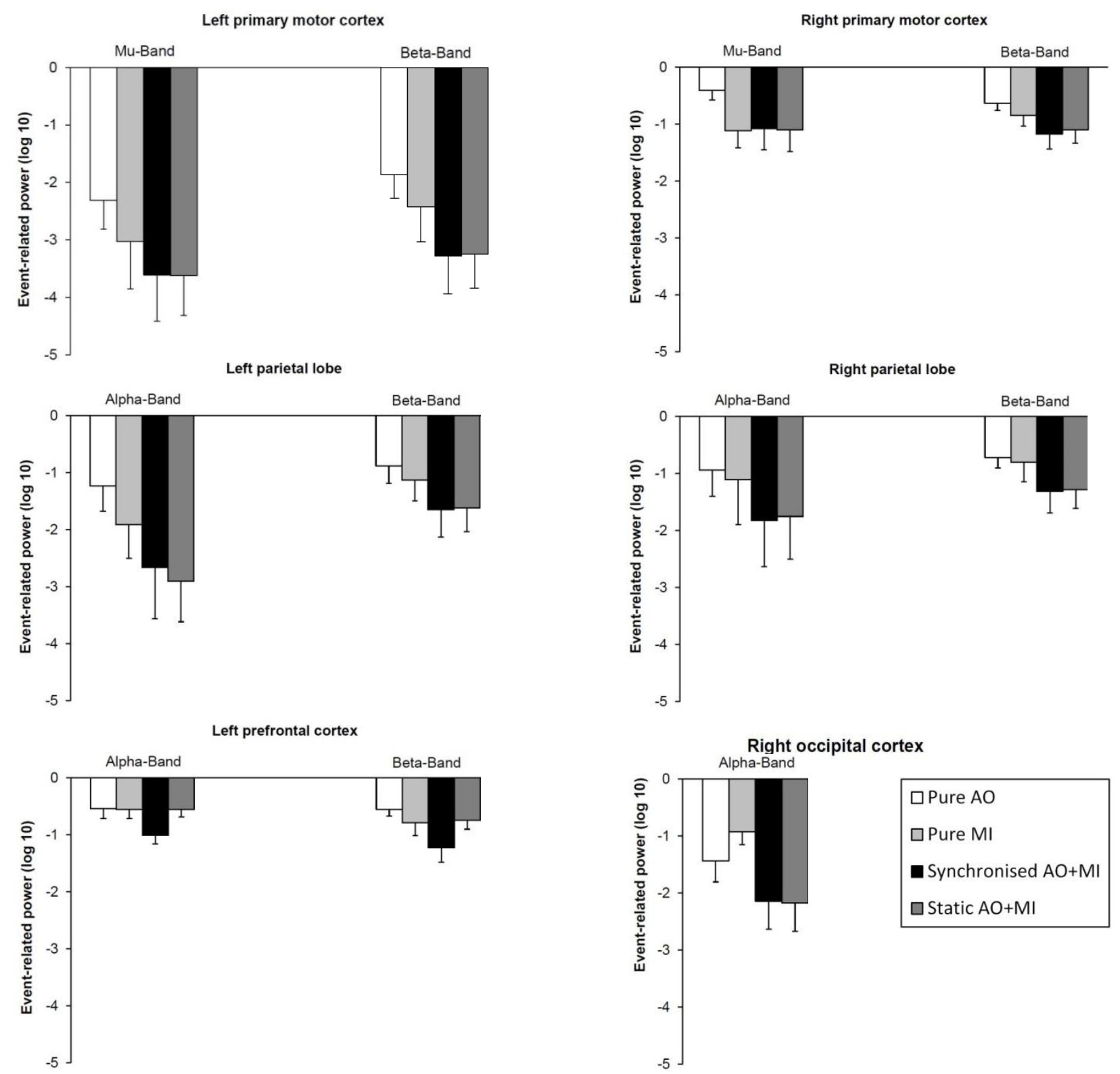


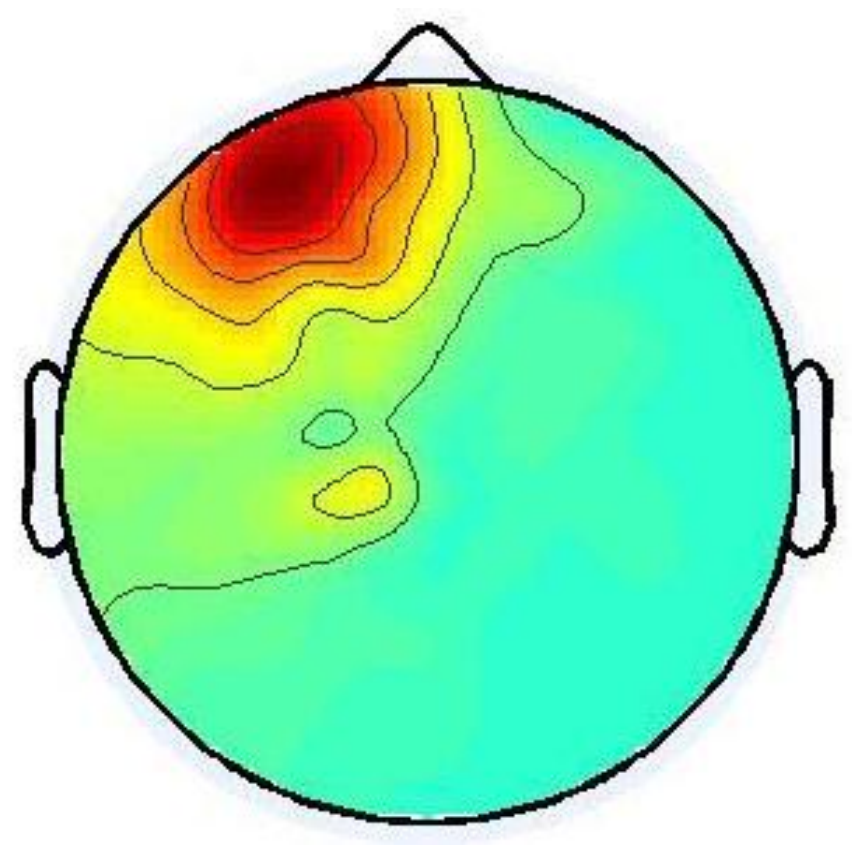

